\title{
INVARIANT DIFFERENTIAL OPERATORS ON A REDUCTIVE LIE ALGEBRA AND WEYL GROUP REPRESENTATIONS
}

\author{
NOLAN R. WALLACH
}

\section{INTRODUCTION}

Let $V$ be a finite-dimensional vector space, and let $G$ be a subgroup of $\mathrm{GL}(V)$. Set $\mathbf{D}(V)$ equal to the algebra of differential operators on $V$ with polynomial coefficients and $\mathbf{D}(V)^{G}$ equal to the $G$ invariants in $\mathbf{D}(V)$. If $\mathfrak{g}$ is a reductive Lie algebra over $C$ then $\mathfrak{h} \subset \mathfrak{g}$ is a Cartan subgroup of $\mathfrak{g}$, and if $G$ is the adjoint group of $\mathfrak{g}$ then $W$ is the Weyl group of $(\mathfrak{g}, \mathfrak{h})$, Harish-Chandra introduced an algebra homomorphism, $\delta$, of $\mathbf{D}(\mathfrak{g})^{G}$ to $\mathbf{D}(\mathfrak{h})^{W}[\mathrm{H} 3] . \delta$ is given by the obvious restriction mapping on the subalgebra of invariant polynomials and on the invariant constant coefficient differential operators, and $\operatorname{ker} \delta$ is the ideal, $\mathscr{I}$, of $\mathbf{D}(\mathfrak{g})^{G}$ consisting of elements that annihilate all $G$ invariant polynomials. In this paper we prove that if $\mathfrak{g}$ has no factor of type $E$ then $\delta$ is surjective. We also prove that for general $\mathfrak{g}$, the homomorphism is surjective after localizing by the discriminant of $\mathfrak{g}$. If $\mathfrak{g}_{o}$ is a real form of $\mathfrak{g}$ and if $G_{o}$ is the adjoint group of $\mathfrak{g}_{o}$ then Harish-Chandra has shown that $\mathscr{I}$ is precisely the ideal in $\mathbf{D}(\mathfrak{g})^{G}$ of operators that annihilate all $G_{o}$ invariant distributions on "completely invariant" open subsets of $\mathfrak{g}_{o}$ [H2]. Our first application of our analysis of $\delta$ is to give a new proof of this important theorem.

In light of this theorem the space of $G_{o}$-invariant distributions on a completely invariant open subset of $\mathfrak{g}_{o}$ is a $\mathbf{D}(\mathfrak{g})^{G}$-module that "pushes down" to a $\mathbf{D}(\mathfrak{h})^{W}$-module. To analyze these $\mathbf{D}(\mathfrak{h})^{W}$-modules we develop a theory analogous to Howe's formalism of dual pairs, proving an equivalence of categories between an appropriate category of $\mathbf{D}(\mathfrak{h})^{W}$-modules and the category of all $W$-modules over $\mathbf{C}$. We show that the $\mathbf{D}(\mathfrak{g})^{G}$-module of distributions on $\mathfrak{g}_{o}$ supported in the nilpotent cone of $\mathfrak{g}_{o}$ is (as a $\mathbf{D}(\mathfrak{h})^{W}$ module) in our category. Thus, to each distribution supported on the nilpotent cone we can associate a (finite dimensional) representation of $W$. If the distribution is the orbital integral corresponding to a fixed nilpotent element of $\mathfrak{g}_{o}$ then we prove that the representation of $W$ is irreducible and derive a formula for the Fourier transform of the orbital integral in terms of $W$-harmonic polynomials corresponding

Received by the editors April 17, 1991 and, in revised form, June 5, 1992.

1991 Mathematics Subject Classification. Primary 22E30, 22E45.

Key words and phrases. Research partially supported by an NSF summer grant. 
to this representation of $W$. In [BV2, BV3, $\mathrm{HK}]$ results of this nature were proved in the case when $\mathfrak{g}_{o}$ is a Lie algebra over $\mathbf{C}$ looked upon as a Lie algebra over $\mathbf{R}$. They prove that the indicated Fourier transform is given in terms of a harmonic polynomial transforming according to the Springer representation associated to the corresponding nilpotent $G$-orbit in $\mathfrak{g}$ [S]. We use this theorem to prove that our general correspondence between nilpotent $G_{o}$-orbits of $\mathfrak{g}_{o}$ is given by the Springer correspondence for the corresponding $G$-orbits in $\mathfrak{g}$. In particular, our theory yields a new approach to the Springer correspondence.

We say that $\mathfrak{g}$ is "nice" if $\delta$ is surjective. (As indicated above one can prove "niceness" for all $\mathfrak{g}$ without ideals of type $E$.) For nice $\mathfrak{g}$ we show that the surjectivity of $\delta$ can be used to give a new proof of Harish-Chandra's famous local $L^{1}$ theorem for invariant eigendistributions on completely invariant subsets of $\mathfrak{g}_{o}$. The question of whether or not $\mathfrak{g}$ is nice is related to a long standing problem concerning Weyl group invariants in two copies of a Cartan subalgebra. We consider the contragradient action of $W$ on $\mathfrak{h}^{*}$ and are interested in the invariants of $W$ in $\mathscr{P}\left(\mathfrak{h} \times \mathfrak{h}^{*}\right)$ (the polynomials on $\left.\mathfrak{h} \times \mathfrak{h}^{*}\right), \mathscr{P}\left(\mathfrak{h} \times \mathfrak{h}^{*}\right)^{W}$, under the action $s f(x, \lambda)=f\left(s^{-1} x, s^{-1} \lambda\right)$. We choose a basis of $\mathfrak{h}$ and the dual basis in $\mathfrak{h}^{*}$ and thereby have linear coordinates $x_{1}, \ldots, x_{1}$ on $\mathfrak{h}$ and dual linear coordinates $\xi_{1}, \ldots, \xi_{l}$ on $\mathfrak{h}^{*}$. Set $P=\sum \xi_{i} \partial / \partial x_{i}$ on $\mathscr{P}\left(\mathfrak{h} \times \mathfrak{h}^{*}\right) \quad(P$ is the usual polarization operator). $P$ stabilizes $\mathscr{P}\left(\mathfrak{h} \times \mathfrak{h}^{*}\right)^{W}$, and it has been suggested that $\mathscr{P}\left(\mathfrak{h} \times \mathfrak{h}^{*}\right)^{W}$ is the algebra generated by $\sum P^{k} \mathscr{P}(\mathfrak{h})^{W}$. For lack of a name let us call this the "polarization hypothesis". If this were true then it is a simple matter to prove that all $\mathfrak{g}$ are good. For $\mathfrak{g}$ of type $A_{n}$ the polarization hypothesis can be found in [W]. By a modification of the argument of Weyl it is easy to show that the hypothesis is also true for types $B_{n}$ and $C_{n}$ and $G_{2}$. However, the hypothesis is false for $D_{n}$ for $n \geq 4$. In the first appendix to this paper we give a counterexample for $D_{4}$ and introduce what we call the "revised polarization hypothesis". This revision is sufficient to prove "niceness", and it is true for $D_{n}$. For $F_{4}$ even this is false. However, one can prove a result for $F_{4}$ which is sufficient to prove that it is also nice. The proof for $F_{4}$ will appear elsewhere. For $E_{6}, E_{7}$, and $E_{8}$ the question of niceness will most likely be testable using the next generation of computers. However, we hope that there is an elegant theorem on Weyl group invariants (in the spirit of Chevalley's proof that $\mathscr{P}(\mathfrak{h})^{W}$ is a polynomial ring) that will give a uniform argument.

The author began his work on this chain of ideas after a conversation he had with Roger Howe (walking in the Torrey Pines Reserve). In this conversation Howe described his work on the action of the algebra generated by the Casimir polynomial and the Laplacian on $\mathscr{D}^{\prime}(\mathfrak{s l}(2, \mathbf{R}))^{G_{o}}$. An outgrowth of this conversation was that it seemed quite likely that $\mathbf{D}(\mathfrak{h})^{W}$ that is generated by the Weyl group invariant polynomials and the Weyl group invariant differential operators (indeed, Howe sketched a proof of the result for $A_{n}$ using a theorem of Weyl alluded to above). We also thank T. Enright, B. Kostant, and D. Vogan for helpful conversations.

\section{POLYNOMIAL DIFFERENTIAL OPERATORS INVARIANT UNDER A FINITE GROUP}

We begin this section with a simple result that will play an important role in this paper. 
Lemma 1.1. Let $\mathscr{A}$ be an algebra over $\mathbf{C}$ with unit, and let $\mathscr{B}$ be a subalgebra of $\mathscr{A}$ containing 1 such that there exists a linear map $P$ of $\mathscr{A}$ onto $\mathscr{B}$ such that $P(1)=1$ and $P(a b)=P(a) b$ for $a \in \mathscr{A}, b \in \mathscr{B}$. If $V$ is $a \mathscr{B}$-module then the map $V \rightarrow \mathscr{A} \otimes_{\mathscr{B}} V$ given by $v \mapsto 1 \otimes_{\mathscr{B}} v$ is injective.

Proof. We denote by $\mathscr{A} \otimes V$ the tensor product over C. Suppose that $v \in V$ and $1 \otimes_{\mathscr{B}} v=0$ in $\mathscr{A} \otimes_{\mathscr{B}} V$. Then there exist $a_{1}, \ldots, a_{m} \in \mathscr{A}, v_{1}, \ldots, v_{n} \in$ $V$, and $b_{i j} \in \mathscr{B}$ such that

$$
1 \otimes v=\sum_{i j} a_{i}\left(b_{i j} \otimes v_{j}-1 \otimes b_{i j} v_{j}\right) .
$$

If we apply $P \otimes I$ to both sides of (1.1) then we have $(P(1)=1)$

$$
1 \otimes v=\sum_{i j} P\left(a_{i}\right)\left(b_{i j} \otimes v_{j}-1 \otimes b_{i j} v_{j}\right) \in \mathscr{B} \otimes V .
$$

Thus $1 \otimes_{\mathscr{B}} v=0$ in $\mathscr{B} \otimes_{\mathscr{B}} V$. Hence $v=0$.

Note. Let $\mathscr{A}$ be an algebra over $\mathbf{C}$ with a filtration $\mathscr{A}^{i} \subset \mathscr{A}^{i+1}, \bigcup_{i} \mathscr{A}^{i}=$ $\mathscr{A}, \operatorname{dim} \mathscr{A}^{i}<\infty$. Let $G$ be a compact Lie group acting on $\mathscr{A}$ by automorphisms such that $g \mathscr{A}^{i} \subset \mathscr{A}^{i}$ for all $i$ and $g \in G$. If the corresponding representation of $G$ on $\mathscr{A}^{i}$ is continuous for all $i$ and if $\mathscr{B}=\mathscr{A}^{G}=\{a \in$ $\mathscr{A} \mid g a=a, g \in G\}$ then set

$$
P a=\int_{G} g(a) d g
$$

with $d g$ normalized invariant measure on $G$. The conclusion of Lemma 1 is therefore true for $\mathscr{B}$. We will apply Lemma 1 to this context without further comment.

Let $V$ be a finite-dimensional vector space over $\mathbf{C}$. We will use the notation in Appendix 1. Let $G$ be a subgroup of $\mathrm{GL}(V)$. Then $G$ acts on $\mathscr{P}(V)$ by $g \cdot f(x)=f\left(g^{-1} x\right)$ for $f \in \mathscr{P}(V), x \in V, g \in G$. If $D \in \mathbf{D}(V)$ then we set $g \cdot \mathbf{D}=g D g^{-1}$. We note that if $g \in G$ then $g \cdot \mathbf{D}^{k}(V) \subset \mathbf{D}^{k}(V)$. If $M$ is a $G$-module then we set

$$
M^{G}=\{m \in M \mid g \cdot m=m, g \in G\} .
$$

Then $\mathscr{P}(V)^{G}, S(V)^{G}$, and $\mathbf{D}(V)^{G}$ are respectively subalgebras of $\mathscr{P}(V), S(V)$, and $\mathbf{D}(V)$. We include the following observation since its proof involves one of the basic ideas in the paper.

Lemma 1.2. If $G$ is a finite group then $\mathbf{D}(V)^{G}$ is a simple algebra over $\mathbf{C}$.

Proof. If $D \in \mathbf{D}(V)$ then we use the notation $\operatorname{ord}(D)$ for the usual order of $D$ as a differential operator. Let $I$ be a nonzero two sided ideal in $\mathbf{D}(V)^{G}$. Let $D \in I$ be a nonzero element with $\operatorname{ord}(D)$ minimal. If $f \in \mathscr{P}(V)^{G}$ then $\operatorname{ord}[f, D]<\operatorname{ord}(D)$. Thus, $[f, D]=0$ for all $f \in \mathscr{P}(V)^{G}$. This implies that $D(f h)=f D h$ for all $f, g \in \mathscr{P}(V)^{G}$. Hence $D$ acts on $\mathscr{P}(V)^{G}$ by 
multiplication by $D \cdot 1 \in \mathscr{P}(V)^{G}$. Since $\mathscr{P}(V)$ is finitely generated as a $\mathscr{P}(V)^{G}$-module under multiplication, there exist $u_{1}, \ldots, u_{n} \in \mathscr{P}(V)^{G}$ such that $d u_{1} \wedge \cdots \wedge d u_{n} \neq 0$. This implies that there is an open nonempty subset, $U$, of $V$ such that $u_{1}, \ldots, u_{n}$ defines a system of holomorphic local coordinates on $U$. Thus if $D_{1} \in \mathbf{D}(V)$ then

$$
D_{1}=\sum_{I} a_{I}\left(u_{1}, \ldots, u_{n}\right) \frac{\partial^{|I|}}{\partial u_{1}^{i_{1}} \cdots \partial u_{n}^{i_{n}}}
$$

on $U$. Since $\mathbf{C}\left[u_{1}, \ldots, u_{n}\right]$ is contained in $\mathscr{P}(V)^{G}$, we see that if $D_{1} \mathscr{P}(V)^{G}=$ 0 then $D_{1}=0$. Hence $D$ is given by multiplication by $f=D \cdot 1$. Since $D \neq 0, f \neq 0$. As a right $\mathbf{D}(V)^{G}$-module under multiplication $\mathbf{D}(V)$ is finitely generated. Hence there exist $D_{1}, \ldots, D_{p}$ such that $\mathbf{D}(V)=\sum D_{i} \mathbf{D}(V)^{G}$. Set $M=\mathbf{D}(V)^{G} / I$. Then $f \cdot M=0$. There exists $k$ such that $(a d f)^{k} D_{j}=0$ for all $1 \leq j \leq p$. Thus $f^{k+1}$ acts by 0 on $\mathbf{D}(V) \otimes_{\mathbf{D}(V)^{G}} M$. Since $\mathbf{D}(V)$ is simple, this implies that $\mathbf{D}(V) \otimes_{\mathbf{D}(V)^{G}} M=0$. So Lemma 1.1 implies that $M=0$. Thus $I=\mathbf{D}(V)^{G}$.

We look upon $\mathscr{P}(V)$ as a $\mathbf{D}(V)$-module under the usual action as differential operators, and we look upon $S(V)$ as a $\mathbf{D}(V)$-module under the obvious identification with $\mathbf{D}(V) / \mathbf{D}(V) \mathscr{P}_{+}(V)$ (notation as in Appendix 1).

Proposition 1.3. Assume that $G$ is finite. Let $M$ be a $\mathbf{D}(V)^{G}$-module such that if $m \in M$ then $\operatorname{dim} S(V)^{G} m<\infty\left(\right.$ resp. $\left.\operatorname{dim} \mathscr{P}(V)^{G} m<\infty\right)$. If $m \in M, p \in$ $\mathscr{P}(V)^{G}-\{0\}$ (resp. $S(V)^{G}-\{0\}$ ) is such that pm $=0$ then $m=0$. If $M$ is finitely generated as a $\mathbf{D}(V)^{G}$-module then $M$ is of finite length.

Proof. We assume that if $m \in M$ then $\operatorname{dim} S(V)^{G} m<\infty$. Let $m \in M$ be such that $p m=0$ for some $p \in \mathscr{P}(V)^{G}-\{0\}$. Set $N=\mathbf{D}(V)^{G} m$. We show that $N=\{0\}$. Set $N_{1}=\mathbf{D}(V) \otimes_{\mathbf{D}(V)}{ }^{G} N$. If $q \in S(V)$ (resp. $f \in \mathscr{P}(V)$ ) and if $D \in \mathbf{D}(V)$ then there exists $k$ such that $a d(q)^{k} D=0$ (resp. $\operatorname{ad}(f)^{k} D=0$ ). Since $S(V)$ is finitely generated as a $S(V)^{G}$-module under multiplication, this implies that if $m_{1}=1 \otimes m$ then $\operatorname{dim} S(V) m_{1}<\infty$. Set $F=S(V) m_{1}$. Then there exists $k$ such that $p^{k} F=0$. Furthermore, $N_{1}=\mathscr{P}(V) F$. We filter $N_{1}$ by setting $\mathscr{F}^{j} N_{1}=\sum_{i \leq j} \mathscr{P}_{j}(V) F$. Then

$$
\mathbf{D}^{i}(V) \mathscr{F}^{j} N_{1} \subset \mathscr{F}^{i+j} N_{1} .
$$

Let $d$ be the degree of $p$. Our assumptions imply that

$$
\operatorname{dim} \mathscr{F}^{j} N_{1} \leq \operatorname{dim} F \sum_{i \leq j}\left(\operatorname{dim} S_{i}(V)-\operatorname{dim} S_{i-d k}(V)\right) \leq C j^{n-1} .
$$

Thus Bernstein's theorem (see Appendix 1, Theorem 2) implies that $N_{1}=\{0\}$. We now assume that $M$ is finitely generated as a $\mathbf{D}(V)^{G}$-module. Set

$$
M_{1}=\mathbf{D}(V) \otimes_{\mathbf{D}(V)^{G}} M
$$


If $z_{1}, \ldots, z_{d}$ are such that $\mathbf{D}(V)=\sum_{i} z_{i} \mathbf{D}(V){ }^{G}$ and if $M=\mathbf{D}(V){ }^{G} F$ with $F$ a finite-dimensional subspace of $M$ then, if $F_{1}=\sum_{i} z_{i} \otimes_{\mathbf{D}(V)^{G}} F, F_{1}$ generates $M_{1}$ as a $\mathbf{D}(V)$-module. As above, $\operatorname{dim} S(V) F_{1}<\infty$. If we filter $M_{1}$ as above then we have a filtration $\mathscr{F}^{k} M_{1}$ of $M_{1}$ so that $M_{1}$ is a filtered module. The argument above implies that

$$
\operatorname{dim} \mathscr{F}^{k} M_{1} \leq C k^{n}
$$

Thus $M_{1}$ is of finite length (see Appendix 1, Theorem 2) as a $\mathbf{D}(V)$-module. We will now show that this implies that $M$ is of finite length as a $\mathbf{D}(V)^{G}$-module. Indeed, let

$$
M \supset M^{1} \supset M^{2} \supset \cdots \supset M^{k} \supset \cdots
$$

be a decreasing family of submodules of $M$. Set $N^{k}$ equal to the canonical image of $\mathbf{D}(V) \otimes_{\mathbf{D}(V)^{G}} M^{k}$ in $M^{1}$. Then $N^{k} \supset N^{k+1}$. Since $M_{1}$ is of finite length, there exists $k$ such that $N^{k}=N^{p}$ for all $p \geq k$. This implies that if $v \in M^{k}$ and if $p \geq k$ then there exist $D_{i}, d_{i} \in \mathbf{D}(V), e_{i} \in \mathbf{D}(V)^{G}, v_{i} \in$ $M^{p}, w_{i} \in M$ such that (the following tensor products are over $\mathbf{C}$ )

$$
1 \otimes v=\sum D_{i} \otimes v_{i}+\sum_{i j l}\left(d_{i} e_{j} \otimes w_{l}-d_{i} \otimes e_{j} w_{l}\right) .
$$

Thus, in the notation of Lemma 1.1, we have

$$
1 \otimes v=\sum P\left(D_{i}\right) \otimes v_{i}+\sum_{i j l}\left(P\left(d_{i}\right) e_{j} \otimes w_{l}-P\left(d_{i}\right) \otimes e_{j} w_{l}\right) .
$$

Hence $v=\sum P\left(D_{i}\right) v_{i} \in M^{p}$. Thus $M^{p} \supset M^{k}$. Hence $M^{p}=M^{k}$ for $p \geq k$. The proof in this case is now complete.

The proof of the parenthetic statements is the same after we have reversed the roles of $\mathscr{P}(V)$ and $S(V)$.

Let $\mathscr{C}_{G}$ (resp. $\mathscr{C}_{G}^{\prime}$ ) denote the category of all finitely generated $\mathbf{D}(V) G$ modules such that if $m \in M$ then there exists $k$ such that if $p \in S_{k}(V)^{G}$ (resp. $\left.p \in \mathscr{P}_{k}(V)\right)$ then $p m=0$. If $G$ leaves invariant a symmetric nondegenerate form and if $\phi$ is as above then the functor $M \rightarrow \widetilde{M}$ defines an equivalence of categories between $\mathscr{C}_{G}$ and $\mathscr{C}_{G}^{\prime}$. The following result is a direct consequence of Proposition 1.3.

Corollary 1.4. Assume then $G$ is finite. If $M \in \mathscr{C}_{G}$ (resp. $C_{G}^{\prime}$ ) and if $m \in$ $M, p \in \mathscr{P}(V)^{G}-\{0\}$ (resp. $S(V)^{G}-\{0\}$ ) is such that $p m=0$ then $m=0$.

We define a $\mathbf{D}(V)$ module structure on $S(V)$ as follows. Let $\mathbf{C}_{0}$ be the $\mathscr{P}(V)$-module, $\mathbf{C}$, with $f \cdot 1=f(0)$. Then as an $S(V)$-module, $\mathbf{D}(V) \otimes_{\mathscr{P}(V)} \mathbf{C}_{0}$ is $S(V) \otimes 1$. We look upon $S(V)$ as a $G$-module in the usual way. Thus with this $\mathbf{D}(V)$-module structure $S(V)$ is a $\left(\mathbf{D}(V)^{G}, G\right)$-bimodule (as is $\left.\mathscr{P}(V)\right)$. This is the desired structure on $S(V) . \mathscr{P}(V) \in \mathscr{C}_{G}$ and $S(V) \in \mathscr{C}_{G}^{\prime}$. 
Proposition 1.5. Assume that $G$ acts completely reducibly on $V$. Let $\mathscr{S}$ (resp. $\widetilde{\mathscr{S}})$ denote the set of all isomorphism classes of irreducible finite-dimensional $G$ modules, $M$, such that $\operatorname{Hom}_{G}(M, \mathscr{P}(V)) \neq 0\left(\right.$ resp. $\left.\operatorname{Hom}_{G}(M, S(V)) \neq 0\right)$. If $\lambda \in \mathscr{S} \cup \widetilde{\mathscr{S}}$ then fix $V_{\lambda} \in \lambda$. To each $\lambda \in \mathscr{S}$ (resp. $\lambda \in \widetilde{\mathscr{S}}$ ) there corresponds an irreducible $\mathbf{D}(V)^{G}$-module $V^{\lambda}$ (resp. $\tilde{V}^{\lambda}$ ) such that:

(i) If $V^{\lambda}$ (resp. $\widetilde{V}^{\lambda}$ ) is equivalent with $V^{\mu}$ (resp. $\widetilde{V}^{\mu}$ ) as a $\mathbf{D}(V)^{G}$-module then $\lambda=\mu$; and

(ii) As a $\left(\mathbf{D}(V)^{G}, G\right)$-bimodule $\mathscr{P}(V)$ (resp. $S(V)$ ) is equivalent with

$$
\bigoplus_{\lambda \in \mathscr{S}} V^{\lambda} \otimes V_{\lambda} \quad\left(\text { resp. } \bigoplus_{\lambda \in \tilde{\mathscr{S}}} \tilde{V}^{\lambda} \otimes V_{\lambda}\right) \text {. }
$$

Proof. If $g \in \mathrm{GL}(V)$, let $g^{t}$ denote the element of $\mathrm{GL}\left(V^{*}\right)$ given by $g^{t} \mu=$ $\mu \circ g^{-1}, \mu \in V^{*}$. Set $G^{t}=\left\{g^{t} \mid g \in G\right\} \subset \mathrm{GL}\left(V^{*}\right)$. Let $x_{1}, \ldots, x_{n}$ be linear coordinates on $V$. Let $\psi$ be the automorphism of $\mathbf{D}(V)$ defined by $\psi\left(x_{i}\right)=\partial_{i}$ and $\psi\left(\partial_{i}\right)=-x_{i}$. Then $\psi\left(\mathbf{D}(V)^{G}\right)=\mathbf{D}(V)^{G^{t}}, \psi(\mathscr{P}(V))=S(V)$, and $\psi(g f)=g^{t} \psi(f)$ for $f \in \mathscr{P}(V), g \in G$. Thus if we prove the result for $\mathscr{P}(V)$ then the result will follow for $S(V)$.

We will be using the following construction throughout the proof of this result. Let $\lambda \in \mathscr{S}$, and let $\lambda^{*}$ be the class of the contragredient representation of $V_{\lambda}, V_{\lambda}^{*}$. Then $\lambda^{*} \in \widetilde{\mathscr{S}}$. Let $e_{1}, \ldots, e_{d}$ be basis of $V_{\lambda}$, and let $e_{1}^{*}, \ldots, e_{d}^{*}$ be the dual basis in $V_{\lambda}^{*}$. If $T \in \operatorname{Hom}_{G}\left(V_{\lambda}, \mathscr{P}(V)\right)$ and $S \in \operatorname{Hom}_{G}\left(V_{\lambda}^{*}, S(V)\right)$ then set $D_{T, S}=\sum_{i} T\left(e_{i}\right) S\left(e_{i}^{*}\right)$. Then $D_{T, S} \in \mathbf{D}(V)^{G}$.

If $\lambda \in \mathscr{S}^{\prime}$ then let $\mathscr{P}(V)[\lambda]$ denote the $\lambda$-isotypic component of $\mathscr{P}(V)$. It is clear that as a $\left(\mathbf{D}(V)^{G}, G\right)$-bimodule $\mathscr{P}(V)$ splits into a direct sum of the invariant subspaces $\mathscr{P}(V)[\lambda]$. Fix $Z_{\lambda}$ a nonzero $G$-invariant irreducible subspace of $\mathscr{P}(V)[\lambda]$. Let $M \subset \mathscr{P}(V)[\lambda]$ be a nonzero $\mathbf{D}(V)^{G}$-invariant subspace. Let $E=\sum_{j} x_{j} \partial_{j}$. Then $E \in \mathbf{D}(V)^{G}$. Thus $E M \subset M$. Hence if $f \in M$ then every homogeneous component of $f$ is in $M$.

(1) $M \cap Z_{\lambda} \neq\{0\}$.

Indeed, let $f \in M-\{0\}$ be homogeneous of degree $r$. Then $\operatorname{span}_{\mathbf{C}}\{g f\}$ is a direct sum of irreducible $G$-submodules in the class $\lambda$. Let $T$ be a nonzero element of $\operatorname{Hom}_{G}\left(V_{\lambda}, Z_{\lambda}\right)$. Let $S \in \operatorname{Hom}_{G}\left(V_{\lambda}^{*}, S_{r}(V)\right)$ be such that there exists $\mu \in V_{\lambda}^{*}$ with $S(\mu) f=1 . S$ and $\mu$ exist since $f$ is homogeneous and the pairing between $S_{r}(V)$ and $\mathscr{P}_{r}(V)$ given by $(p \mid f)=p f \in \mathbf{C}$ is perfect. Fix a basis $e_{1}, \ldots, e_{d}$ of $V_{\lambda}$ such that $e_{1}^{*}=\mu$ and $S\left(e_{i}^{*}\right) f=0$ for $i>1$. Then $D_{T, S} f=T\left(e_{1}\right)$. This implies (1).

Let $f \in Z_{\lambda}$. Put $M=\mathbf{D}(V)^{G} f$. We use the notation $\mathbf{C}[G]$ for the group algebra of $G$ thought of as an abstract group.

(2) $M \cap Z_{\lambda}=\mathbf{C} f$.

Indeed, if $g \in M \cap Z_{\lambda}$ then there exists $D \in \mathbf{D}(V)^{G}$ such that $D f=g$. Thus $\mathbf{C}[G] g=D \mathbf{C}[G] f$. So $D_{\mid Z_{\lambda}} \in \operatorname{Hom}_{G}\left(Z_{\lambda}, Z_{\lambda}\right)$. Schur's lemma implies that $D_{\mid Z_{\lambda}}=\mathbf{C I}$. Thus $g \in \mathbf{C} f$. 
(3) $M$ is irreducible as a $\mathbf{D}(V)^{G}$-module.

In fact, if $M_{1}$ is a nonzero $\mathbf{D}(V)^{G}$-invariant subspace of $V$ then $M_{1} \cap Z_{\lambda} \neq 0$ by (1). Hence $f \in M_{1}$ by (2). Thus $M_{1}=M$.

Let $f_{1}, \ldots, f_{d}$ be a basis of $Z_{\lambda}$. Set $V_{i}=\mathbf{D}(V)^{G} f_{i}$. We assert that the sum $V_{1}+\cdots+V_{d}$ is direct. Let $u_{i k} \in \mathbf{C}[G]$ be such that $u_{i k} f_{l}=\delta_{l k} f_{i}$ (this is possible since $\left.\mathrm{C}[G]_{\mid Z_{\lambda}}=\operatorname{End}\left(Z_{\lambda}\right)\right)$. Let $v_{i} \in V_{i}$, and assume that $\sum_{i} v_{i}=0$. Then $v_{i}=D_{i} f_{i}$ with $D_{i} \in \mathbf{D}(V)^{G}$. Thus

$$
0=u_{i i}\left(v_{1}+\cdots+v_{d}\right)=\sum_{k} D_{k} u_{i i} f_{k}=D_{i} f_{i}=v_{i} .
$$

Similarly, $u_{i j}$ defines a $\mathbf{D}(V)^{G}$-module isomorphism of $V_{j}$ onto $V_{i}$. Let $V^{\lambda}=$ $\mathbf{D}(V)^{G} f$ for some $f \in Z_{\lambda}-\{0\}$. Then since there exists $u \in \mathbf{C}[G]$ with $u f_{1}=f, V_{1}$ is isomorphic with $V^{\lambda}$ as a $\mathbf{D}(V)^{G}$-module. This implies that $\mathscr{P}(V)[\lambda]$ is isomorphic with $V^{\lambda} \otimes V_{\lambda}$ as a $\left(\mathbf{D}(V)^{G}, G\right)$-bimodule. This proves (ii).

If $\lambda \in \mathscr{S}$ then let $j(\lambda)$ be the minimum of $j$ such that $\mathscr{P}_{j}(V) \cap \mathscr{P}(V)[\lambda] \neq$ $\{0\}$. Suppose that $V^{\lambda}$ is equivalent with $V^{\mu}$ with $\lambda, \mu \in \mathscr{S}$. We take these modules to be realized as above. Let $A$ implement the equivalence. Let $j=j(\lambda)$. Since $A E=E A, A\left(V^{\lambda} \cap \mathscr{P}_{j}(V)\right) \subset V^{\mu} \cap \mathscr{P}_{j}(V)$. We may assume that $Z_{\lambda} \subset \mathscr{P}_{j}(V)$. Let $T \in \operatorname{Hom}_{G}\left(V_{\lambda}, Z_{\lambda}\right)$ be nonzero, and let $\mathscr{S} \in$ $\operatorname{Hom}_{G}\left(V_{\lambda}^{*}, S_{j}(V)\right)$ be such that $S\left(V_{\lambda}^{*}\right) Z_{\lambda} \neq 0$. Set $D=D_{T, S}$. Then $D\left(V^{\lambda} \cap\right.$ $\left.\mathscr{P}_{j}(V)\right) \neq 0$. Thus

$$
0 \neq A\left(D\left(V^{\lambda} \cap \mathscr{P}_{j}(V)\right)\right)=D A\left(V^{\lambda} \cap \mathscr{P}_{j}(V)\right) \subset V^{\mu} \cap \mathscr{P}_{j}(V) .
$$

But the formula for $D$ implies that $D \mathscr{P}_{j}(V) \subset Z_{\lambda}$. Thus $V^{\mu} \cap Z_{\lambda} \neq 0$. This implies that $\lambda=\mu$. This completes the proof of (i) and, hence, of the proposition.

Theorem 1.6. Assume that $G$ is finite. Then $\mathscr{S}=\widehat{G}$. If $\lambda \in \widehat{G}$, let $V^{\lambda} \in \mathscr{C}_{G}$ (resp. $\widetilde{V}^{\lambda} \in \mathscr{C}_{G}^{\prime}$ ) be as in Proposition 1.5 .

(i) If $M \in \mathscr{C}_{G}$ (resp. $\mathscr{\mathscr { C }}_{G}^{\prime}$ ) and $V$ is irreducible then $M$ is equivalent with $V^{\lambda}\left(\right.$ resp. $\left.\widetilde{V}^{\lambda}\right)$ for some $\lambda \in \widehat{G}$.

(ii) If $M \in \mathscr{C}_{G}$ (resp. $M \in \mathscr{C}_{G}^{\prime}$ ) then for each $\lambda \in \widehat{G}$ there exists $m_{\lambda} \in \mathbf{N}$ such that $M$ is equivalent with

$$
\bigoplus_{\lambda \in \widehat{G}} m_{\lambda} V^{\lambda} \quad\left(\text { resp. } \bigoplus_{\lambda \in \widehat{G}} m_{\lambda} \widetilde{V}^{\lambda}\right) .
$$

That is, every object in $\mathscr{C}_{G}$ (resp. $\mathscr{\mathscr { C }}_{G}^{\prime}$ ) splits into irreducible components with finite multiplicities.

Proof. As above it is enough to prove the result in the case of $\mathscr{C}_{G}$. For the sake of completeness, we give the standard proof that $\mathscr{S}=\widehat{G}$. If $g \in G$ and $g \neq I$ then set $U_{g}=\{v \in V \mid g v \neq v\}$. Then $V-U_{g}=\operatorname{ker}(g-I)$ 
which is a proper subspace of $V$ if $g \neq I$. Since $G$ is finite, this implies that $V^{\prime}=\bigcap_{g \in G-\{I\}} U_{g} \neq \varnothing$. Fix $v \in V^{\prime}$. Set $\mathscr{I}=\{f \in \mathscr{P}(V) \mid f(G v)=0\}$. Then $\mathscr{I}$ is $G$-invariant and $\mathscr{P}(V) / \mathscr{I}$ is equivalent with $\mathbf{C}[G]^{*} \cong \mathbf{C}[G]$ as a $G$-module.

(1) If $M \in \mathscr{C}_{G}$ then $\mathbf{D}(V) \otimes_{\mathbf{D}(V)^{G}} M \in \mathscr{C}\left(=\mathscr{C}_{\{1\}}\right)$.

Indeed, we set $U=\mathbf{D}(V) \otimes_{\mathbf{D}(V)^{G}} M$. We note that as a $\mathbf{D}(V)^{G}$-module $U \in \mathscr{C}_{G}$. We must show that if $p \in S_{+}(V)$ and $m \in U$ then there exists $k$ such that $p^{k} m=0$. Since $S(V)$ is integral over $S(V)^{G}$, there exist $j>0$ and $a_{o}, \ldots, a_{j-1} \in S(V)^{G}$ such that

$$
p^{j}+\sum_{i=0}^{j-1} p^{i} a_{i}=0 .
$$

Clearly, we may assume that $a_{i} \in S_{+}(V)^{G}$. Let $m \in U$. Set $Z=S(V)^{G} m$. Then $Z_{1}=Z+p Z+\cdots+p^{j-1} Z$ is $p$-invariant. Since $S(V)$ is commutative, $Z_{1}$ is $S(V)^{G}$-invariant. Let $Z_{1}^{0}=\left\{z \in Z_{1} \mid a_{i} z=0, i=1, \ldots, j-1\right\} \quad Z_{1}^{1}=$ $\left\{z \in Z_{1} \mid a_{r} a_{s} z=0, r, s \leq j-1\right\}, \ldots$. Then since $Z_{1}$ is finite dimensional, we have $Z_{1}^{0} \subset Z_{1}^{1} \subset \cdots \subset Z_{1}^{q}=Z_{1}$. Clearly, $p Z_{1}^{i} \subset Z_{1}^{i}$. (*) implies that $p^{j} Z_{1}^{0}=0$. We note that $a_{i} Z_{1}^{i} \subset Z_{1}^{i-1}$. Thus $p^{j} Z_{1}^{i} \subset Z_{1}^{i-1}$ (by (*)). Hence $p^{j(q+1)} Z_{1}^{q}=0$. Hence $p^{j(q+1)} m=0$.

Lemma 1.3 now implies that $\mathbf{D}(V) \otimes_{\mathbf{D}(V)^{G}} M$ is a finite multiple of $\mathscr{P}(V)$ as a $\mathbf{D}(V)$-module. Lemma 1.1 implies that $M$ injects in $\mathbf{D}(V) \otimes_{\mathbf{D}(V)^{G}} M$ as a $\mathbf{D}(V)^{G}$-submodule. Thus Proposition 1.6(ii) implies that $M$ has a decomposition as in (ii). Since (i) is a consequence of (ii), the theorem follows.

\section{DifFERENTIAL OPERATORS INVARIANT UNDER A WEYL GROUP}

Let $V_{o}$ be a real $n$-dimensional vector space over $\mathbf{R}$ with inner product $\langle\ldots, \ldots\rangle$. Let $V=\left(V_{o}\right)_{\mathbf{C}}=V_{o} \otimes_{\mathbf{R}} \mathbf{C}$. We will denote the Hermitian extension of $\langle\ldots, \ldots\rangle$ by the same symbol. Let $\Phi$ be a (reduced) root system contained in $V_{o}^{*}$, and let $W \subset O\left(V_{o}\right)$ be the (finite) group generated by the reflections about the hyperplanes $\alpha=0, \alpha \in \Phi$. Let $u_{1}, \ldots, u_{n}$ be a set of basic homogeneous invariants which we take to be real valued on $V_{o}$. Fix $\Phi^{+}$, a system of positive roots for $\Phi$. Let $x_{1}, \ldots, x_{n}$ be a set of linear coordinates on $V_{o}$ corresponding to an orthonormal basis. Let $\xi_{1}, \ldots, \xi_{n}$ be the dual coordinates on $V_{o}^{*}$. If $f, g \in \mathscr{P}\left(V_{o} \times V_{o}^{*}\right)$ then let $\{f, g\}$ (the Poisson bracket of $f$ and $g$ ) be as in Appendix 1 . We say that the pair $(W, V)$ is "good" if the smallest subalgebra of $\mathscr{P}\left(V \times V^{*}\right)$ containing $\mathscr{P}(V)^{W}$ and $\mathscr{P}\left(V^{*}\right)^{W}$ and closed under $\{\ldots, \ldots\}$ is $\mathscr{P}\left(V \times V^{*}\right)^{W}$ (here $W$ acts under the diagonal action $\left.s(x, \lambda)=\left(s x, \lambda \circ s^{-1}\right)\right)$.

Note that if $V_{o}=V_{o}^{1} \oplus \cdots \oplus V_{o}^{d}$ with $W$ acting irreducibly on $V_{o}^{i}$ then $W$ is equal to $W^{1} \times \cdots \times W^{d}$ with $W^{i}$ a finite subgroup of $V_{o}^{i}$ generated by reflections. Set $V^{i}$ equal to the complexification of $V_{o}^{i}$. Then $\mathbf{D}(V)^{W}$ is equal 
to $\mathbf{D}\left(V^{1}\right)^{W^{1}} \otimes \cdots \otimes \mathbf{D}\left(V^{d}\right)^{W^{d}}$ and $\mathscr{P}(V)^{W}, S(V)^{W}$ split into corresponding tensor products. Also, the Poisson bracket "splits" in a manner consistent with the above direct sum decomposition. We therefore see that if $\left(W^{i}, V^{i}\right)$ is good for $i=1, \ldots, d$ then $(W, V)$ is good.

Proposition 2.1. If $(W, V)$ is good then $\mathbf{D}(V)^{W}$ is the algebra generated by $\mathscr{P}(V)^{W}$ and $S(V)^{W}$ in $\mathbf{D}(V)$.

Proof. We denote by $\mathscr{B}$ the algebra generated by $\mathscr{P}(V)^{W}$ and $S(V)^{W}$ in $\mathbf{D}(V)$. We set $\mathscr{B}^{k}=\mathscr{B} \cap \mathbf{D}^{k}(V)$ and $\mathbf{D}^{k}(V)^{W}=\mathbf{D}^{k}(V) \cap \mathbf{D}(V)^{W}$ (here we are using the notation of Appendix 1). $\sigma$ defines an isomorphism of $\operatorname{Gr} \mathbf{D}(V)$ onto $\mathscr{P}\left(V \times V^{*}\right) . W \times W$ acts on $V_{o} \times V_{o}^{*}$ as a finite group generated by reflections under $(s, t)(v, \lambda)=\left(s v, \lambda \circ t^{-1}\right)$, and $W$ acts on $V_{o} \times V_{o}^{*}$ by the diagonal action (as above). We identify $W$ with the subgroup $\{(s, s) \mid s \in W\} \subset W \times W$. Under these identifications $\operatorname{Gr} \mathbf{D}(V)^{W}=\mathscr{P}\left(V \times V^{*}\right)^{W}$. We set $B=\operatorname{Gr} \mathscr{B}$. Then $B$ is a subalgebra of $\mathscr{P}\left(V \times V^{*}\right)^{W}$ containing $\mathscr{P}(V)^{W}, \mathscr{P}\left(V^{*}\right)^{W}$ and closed under $\{\ldots, \ldots\}$ (see the formula for the "top order symbol" of $\left[D_{1}, D_{2}\right]$ in Appendix 1). Since $(W, V)$ is good, $B \supset \mathscr{P}\left(V \times V^{*}\right)^{W}$. Hence $B=$ $\mathscr{P}\left(V \times V^{*}\right) W$. So $\mathscr{B}=\mathbf{D}(V)^{W}$.

Theorem 2.2. If $\Phi$ has no irreducible component isomorphic with the Weyl group of $E_{6}, E_{7}$, or $E_{8}$ then $(W, V)$ is good. In particular, the conclusion of Proposition 2.1 holds.

Proof. We need only show that if $(W, V)$ is irreducible and not of type $E$ then $(W, V)$ is nice. If $(W, V)$ is not of type $F_{4}$ this follows from Propositions 2 of Appendix 2 since (in the notation of that appendix) $P f=-\frac{1}{2}\{u, f\}$ with $u=\sum_{i} \xi_{i}^{2}, P_{j} f=-\left\{\varphi_{i}(\xi), f\right\}$. In the case of $F_{4}$ the proof of "goodness" is somewhat complicated and will be given elsewhere.

For the rest of this section we will study the module theory of the algebra, $\mathscr{B}$, generated by $\mathscr{P}(V)^{W}$ and $S(V)^{W}$. Let $x_{1}, \ldots, x_{n}$ be linear coordinates on $V_{o}$ corresponding to an orthonormal basis. We define a conjugate linear anti-automorphism $D \mapsto D^{\#}$ of $\mathbf{D}(V)$ by $x_{i} \mapsto \partial_{i}, \partial_{i} \mapsto x_{i}$. If $f, g \in \mathscr{P}(V)$ then we set $\langle f, g\rangle=g^{\#} f(0) .\langle\ldots, \ldots\rangle$ is an inner product on $\mathscr{P}(V)$. If $D \in \mathbf{D}(V)$ then $\langle D f, g\rangle=\left\langle f, D^{\#} g\right\rangle$.

Let $\pi$ be the product of a system of positive roots for $\Phi$. We observe that $\pi^{2} \in \mathscr{P}(V)^{W}$.

Proposition 2.3. There exists $k_{o}$ such that $\pi^{2 k_{o}} \mathbf{D}(V)^{W} \subset \mathscr{B}$.

To prove this result we will introduce some notation and results that will be used throughout this paper. Chevalley's theorem implies that we can choose $u_{1}, \ldots, u_{n}$ with $u_{i}$ homogeneous of degree $d_{i}, d_{i} \leq d_{i+1}$ such that $\operatorname{det}\left[\partial_{i} u_{j}\right]=$ $\pi$. Let $S_{+}(V)$ denote the ideal of elements of $S(V), p$, such that $p 1=0$. Put $S_{+}(V)^{W}=S_{+}(V) \cap S(V)^{W}$. We set $\mathscr{H}=\mathscr{H}(V)=\{f \in \mathscr{P}(V) \mid p f=0, p \in$ 
$\left.S_{+}(V)^{W}\right\}$. Then the map

$$
\mathscr{P}(V)^{W} \otimes \mathscr{H} \rightarrow \mathscr{P}(V)
$$

given by $f \otimes h \mapsto f h$ is a linear bijection. We note that the $u_{j}$ can be chosen such that $u_{j}$ are real valued on $V_{o}$ and such that if $p \in S_{+}(V)^{W}$ and $\operatorname{deg} p<d_{j}$ then $p u_{j}=0$.

Let $\widehat{W}$ denote the set of equivalence classes of irreducible representation of $W$. If $\lambda \in \widehat{W}$, fix $V_{\lambda} \in \lambda$. We look upon $\operatorname{Hom}_{W}\left(V_{\lambda}, \mathscr{P}(V)\right)$ as a $\mathscr{P}(V)^{W}$ module under the action $f T(v)(x)=f(x) T v(x)$. Then $\operatorname{Hom}_{W}\left(V_{\lambda}, \mathscr{P}(V)\right)$ is a free $\mathscr{P}(V)^{W}$-module on generators $\operatorname{Hom}_{W}\left(V_{\lambda}, \mathscr{H}\right)$. Furthermore, $\operatorname{dim} \operatorname{Hom}_{W}\left(V_{\lambda}, \mathscr{H}\right)=\operatorname{dim} V_{\lambda}$.

It is easy to see that it is enough to prove Proposition 2.1 under the assumption that the action of $W$ on $V$ is irreducible. We define $T_{j}\left(x_{i}\right)=\partial_{i} u_{j}$. Then

$$
\operatorname{Hom}_{W}\left(V^{*}, \mathscr{P}(V)\right)=\bigoplus_{j} \mathscr{P}(V)^{W} T_{j}
$$

(1) If $D \in \mathbf{D}(V)^{W}$ is of order (as a differential operator) at most 1 then $D \in \mathscr{B}$.

Indeed, $D 1 \in \mathscr{P}(V)^{W} \subset \mathscr{B}$. Thus replacing $D$ with $D-D 1$ we may assume that $D 1=0$. This implies that $D$ is completely determined by its restriction to $V^{*}$. We therefore see that $D_{\mid V^{*}}=\sum_{i} \varphi_{i} T_{i}$ with $\varphi_{i} \in \mathscr{P}(V)^{W}$. Thus if we define $D_{i}=\sum_{j}\left(\partial_{j} u_{i}\right) \partial_{j}$ then $D=\sum_{i} \varphi_{i} D_{i}$. We set $\Delta=\sum \partial_{i}^{2}$. Then

$$
\left[\Delta, u_{i}\right]=2 D_{i}+\Delta u_{i}
$$

So $D_{i} \in \mathscr{B}$. Hence $D \in \mathscr{B}$ as was to be proved.

(2) If $D \in \mathbf{D}(V)^{W}$ then there exists $k$ such that $\pi^{2 k} D \in \mathscr{B}$.

The proof of this assertion will take some preparation. If $f$ is a polynomial in indeterminates $y_{1}, \ldots, y_{n}$ then

$$
\frac{\partial}{\partial x_{i}} f\left(u_{1}, \ldots, u_{n}\right)=\sum_{j} \frac{\partial f}{\partial y_{j}}\left(u_{1}, \ldots, u_{n}\right) \frac{\partial u_{j}}{\partial x_{i}}
$$

Set $\left[a_{i j}\right]$ equal to the inverse to the matrix $\left[\partial_{i} u_{j}\right]$. Then $\pi a_{i j} \in \mathscr{P}(V)$. Set $\tilde{\delta}_{j}=\sum_{i} a_{i j} \partial_{i}$. Then $\delta_{j}=\pi^{2} \tilde{\delta}_{j} \in \mathbf{D}(V)^{W}$ is of order 1 . Hence $\delta_{j} \in \mathscr{B}$. We note that

$$
\delta_{j} f\left(u_{1}, \ldots, u_{n}\right)=\pi^{2} \frac{\partial f}{\partial y_{j}}\left(u_{1}, \ldots, u_{n}\right) .
$$

Let $x \in V_{o}$ be such that $\pi(x) \neq 0$. Let $U$ be an open connected neighborhood of $x$ in $\mathfrak{h}_{\mathbf{R}}$ such that $u_{1}, \ldots, u_{n}$ define local coordinates on $U$. If $D$ is a differential operator on $U$ with ${ }^{n}{ }^{\infty}$ coefficients then we can write

$$
D=\sum_{i_{1}, \ldots, i_{n}} a_{i_{1}}, \ldots, i_{i_{n}}\left(u_{1}, \ldots, u_{n}\right) \frac{\partial^{i_{1}+\cdots+i_{n}}}{\partial u_{1}^{i_{1}} \cdots \partial u_{n}^{i_{n}}} .
$$


If $D \in \mathbf{D}(V)^{W}$ then $D \mathbf{C}\left[u_{1}, \ldots, u_{n}\right] \subset \mathbf{C}\left[u_{1}, \ldots, u_{n}\right]$. Thus $a_{i_{1}}, \ldots,{ }_{i_{n}}$ is a polynomial. Thus if $D \in \mathbf{D}(V)^{W}$ has order $k$ then there exist polynomials $b_{i_{1} ; \ldots, i_{n}}$ in $n$ indeterminates such that

$$
\pi^{2 k} D=\sum b_{i_{1} \cdots i_{n}}\left(u_{1}, \ldots, u_{n}\right) \delta_{1}^{i_{1}} \cdots \delta_{n}^{i_{n}}
$$

on $U$ and hence, on all of $V_{o}$. This implies (2).

If we use the Bernstein filtration on $\mathbf{D}(V)$ (see Appendix 1) then the corresponding graded ring is $\mathscr{P}\left(V \times V^{*}\right)$. The subring corresponding to the induced grade of $\mathbf{D}(V)^{W}$ is $\mathscr{P}\left(V \times V^{*}\right)^{W}$ relative to the diagonal action of $W$ on $V \times V^{*}$. The Chevalley theorems applied to $W \times W$ imply that $\mathscr{P}\left(V \times V^{*}\right)^{W}$ is free as a $\mathscr{P}\left(V \times V^{*}\right)^{W \times W}$-module on generators $\left(\mathscr{H}(V) \otimes \mathscr{H}\left(V^{*}\right)\right)^{W}$. Let $E$ be a filtered subspace of $\mathbf{D}(V)^{W}$ such that the corresponding graded subspace of $\mathscr{P}\left(V \times V^{*}\right)^{W}$ is $\left(\mathscr{H}(V) \otimes \mathscr{H}\left(V^{*}\right)\right)^{W}$. Then it is easily seen that as a $\left(\mathscr{P}(V)^{W}, S(V)^{W}\right)$-bimodule $\left(\mathscr{P}(V)^{W}\right.$ acts by multiplication on the left and $S(V)^{W}$ acts by multiplication on the right), $\mathbf{D}(V)^{W}$ is isomorphic under the obvious map (multiplication) with $\mathscr{P}(V)^{W} \otimes E \otimes S(V)^{W}$. Since $\operatorname{dim} E<\infty$, (2) implies that there exists $k_{o}$ such that $\pi^{2 k_{o}} E \subset \mathscr{B}$. Hence $\pi^{2 k_{o}} \mathbf{D}(V)^{W} \subset \mathscr{B}$. This completes the proof of Proposition 2.3.

If $\lambda \in \widehat{W}$, let $\mathscr{P}(V)[\lambda]$ denote the $\lambda$-isotypic component of $\mathscr{P}(V)$ with respect to the action of $W$. In Proposition 1.5 we have seen that for each $\lambda \in \widehat{W}$ there exists an irreducible $\mathbf{D}(V)^{W}$-module, $V^{\lambda}$, such that as a $\left(\mathbf{D}(V)^{W}, W\right)$ bimodule,

$$
\mathscr{P}(V) \simeq \bigoplus_{\lambda \in \widehat{W}} V^{\lambda} \otimes V_{\lambda} .
$$

Furthermore, $\mathscr{P}(V)[\lambda] \simeq V^{\lambda} \otimes V_{\lambda}$. The module $V^{\lambda}$ can be realized as follows. Let $Z \subset \mathscr{P}(V)$ be a $W$-invariant irreducible subspace in the class of $\lambda$. Let $h \in Z$ be nonzero. Then $\mathbf{D}(V)^{W} h$ is an irreducible $\mathbf{D}(V)^{W}$ module isomorphic with $V^{\lambda}$. Furthermore,

(3) $\mathbf{D}(V)^{W} h \cap Z=\mathbf{C} h$.

Theorem 2.4. If $\lambda \in \widehat{W}$ then $\mathscr{B}$ acts irreducibly on $V^{\lambda}$.

Proof. Fix $Z$ as above and realize $V^{\lambda}$ as $\mathbf{D}(V)^{W} h=N$. Let $\mathscr{P}(V)_{\left[\pi^{2}\right]}$ denote the subalgebra of the algebra of rational functions on $V$ with denominator powers of $\pi^{2 k}$. That is, $\mathscr{P}(V)_{\left[\pi^{2}\right]}$ is the algebra of functions generated by $x_{1}, \ldots, x_{n}, \pi^{-2}$. Let $N_{\left[\pi^{2}\right]}$ be the subspace of $\mathscr{P}(V)_{\left[\pi^{2}\right]}$ given by $\mathbf{C}\left[\pi^{-2}\right] N$. Let $\mathbf{D}(V)_{\left[\pi^{2}\right]}^{W}$ (resp. $\mathscr{B}_{\left[\pi^{2}\right]}$ ) be the algebra of operators generated by $\mathbf{D}(V)^{W}$ (resp. $\mathscr{B}$ ) and $\pi^{-2}$. Then it is easy to see that $N_{\left[\pi^{2}\right]}$ is a $\mathbf{D}(V)_{\left[\pi^{2}\right]}^{W}$-submodule of $\mathscr{P}(V)_{\left[\pi^{2}\right]}$. Also one can see that if $D \in \mathbf{D}(V)_{\left[\pi^{2}\right]}^{W}$ then there exists $k$ such that $\pi^{2 k} D \in \mathbf{D}(V)^{W}$. Proposition 2.3 implies that

(i) $\mathbf{D}(V)_{\left[\pi^{2}\right]}^{W}=\mathscr{B}_{\left[\pi^{2}\right]}$, and 
(ii) $N_{\left[\pi^{2}\right]}$ is irreducible as a $\mathbf{D}(V)_{\left[\pi^{2}\right]}^{W}$-module.

Indeed, let $M \subset N_{\left[\pi^{2}\right]}$ be a nonzero $\mathbf{D}(V)_{\left[\pi^{2}\right]^{-}}^{W}$-submodule. Let $m \in M$ be nonzero. Then there exists $k$ such that $\pi^{2 k} m \in N$. Thus $\mathbf{D}(V){ }^{W} \pi^{2 k} m=N$. Hence $M=N_{\left[\pi^{2}\right]}$.

Let $M$ be a nonzero $\mathscr{B}$-submodule of $N$. If $m / \pi^{2 k} \in M_{\left[\pi^{2}\right]}$ and if $D \in \mathscr{B}$ then $D\left(m / \pi^{2 k}\right)=\pi^{-2 r} D^{\prime} m$ with $D^{\prime} \in \mathbf{D}(V)^{W}$ and some $r$. There exists $p$ such that $\pi^{2 p} D^{\prime} \in \mathscr{B}$. Hence $D\left(m / \pi^{2 k}\right) \in M_{\left[\pi^{2}\right]}$. Thus $M_{\left[\pi^{2}\right]}$ is a $\mathscr{B}_{\left[\pi^{2}\right]}=$ $\mathbf{D}(V)_{\left[\pi^{2}\right]}^{W}$-submodule of $N_{\left[\pi^{2}\right]}$. So $M_{\left[\pi^{2}\right]}=N_{\left[\pi^{2}\right]}$. This implies

(iii) If $M \subset N$ is a nonzero $\mathscr{B}$-submodule and if $v \in N$ then there exists $k$ such that $\pi^{2 k} v \in M$.

Let $M$ be a nonzero $\mathscr{B}$-submodule of $N$.

(iv) As a $\mathscr{P}(V)^{W}$-module $N$ (resp. $M$ ) is free on generators any basis of $N \cap \mathscr{H}$ (resp. $M \cap \mathscr{H}$ ).

In light of the Chevalley theorems (described above) it is enough to show that $N=\mathscr{P}(V)^{W}(V \cap \mathscr{H})$ (resp. $\left.M=\mathscr{P}(V)^{W}(M \cap \mathscr{H})\right)$. Let $B$ denote either $N$ or $M$. Let $\mathscr{P}^{j}(V)$ denote the space of homogeneous polynomials of degree $j$. Set $B^{j}=B \cap \mathscr{P}^{j}(V)$. Since the Euler operator $\sum x_{i} \partial_{i} \in \mathscr{B}$ (see (1) above), $B$ is the direct sum of the $B^{j}$. Let $j_{o}$ be minimal such that $B^{j_{o}} \neq 0$. If $p \in S_{+}(V)^{W}$ then $p B^{j_{o}} \subset \sum_{i<j_{o}} B^{j}=0$. Thus $B^{j_{o}} \subset \mathscr{H} \cap B$. Assume that we have shown that $B^{j} \subset \mathscr{P}(V)^{W}(B \cap \mathscr{H})$ for $j_{o} \leq j<k$. If $v \in B^{k}$ and $\left\langle v, \mathscr{P}(V)^{W}(B \cap \mathscr{H})\right\rangle=0$ then for $f \in \mathscr{P}(V)^{W}, f(0)=0$ we have

$$
\left\langle v, f\left(\sum_{j<k} B^{j}\right)\right\rangle=0 .
$$

But, $f^{\#} \in S_{+}(V)^{W}$ and $f^{\#} v \in \sum_{j<k} B^{j}$. Hence,

$$
0=\left\langle v, f\left(\sum_{j<k} B^{j}\right)\right\rangle=\left\langle f^{*} v, \sum_{j<k} B^{j}\right\rangle .
$$

So $f^{\#} v=0$. Since $f^{\#}$ is a typical element of $S_{+}(V)^{W}, v \in \mathscr{H} \cap B$. Hence $\langle v, v\rangle=0$. So $v=0$. Thus $B^{k} \subset \mathscr{P}(V)^{W}(B \cap \mathscr{H})$.

We are now ready to complete the proof of the theorem. Let $h_{1}, \ldots, h_{m}$ be a basis of $\mathscr{H} \cap N$ such that $h_{1}, \ldots, h_{r}$ is a basis of $M \cap \mathscr{H}$. If $v \in N$ then $v$ can be written uniquely in the form $v=\sum f_{i} h_{i}$ with $f_{i} \in \mathscr{P}(V)^{W}$. (iii) implies that there exists $k$ such that $\pi^{2 k} v \in M$; thus, (iv) implies that $\pi^{2 k} v=\sum_{i<r} g_{i} h_{i}$ with $g_{i} \in \mathscr{P}(V)^{W}$. Applying (iv) again we find that $\pi^{2 k} f_{i}=0$ for $i>r$. Hence $v \in M$. So $M=N$.

Let $\check{M}$ denote the full subcategory of all $\mathscr{B}$-submodules, $M$, such that $\pi^{2}$ acts injectively on $M$.

Lemma 2.5. If $M \in \check{\mathscr{M}}$ then the map $M \rightarrow \mathbf{D}(V)^{W} \otimes_{\mathscr{B}} M$ given by $m \mapsto 1 \otimes_{\mathscr{B}} m$ is injective. 
Proof. Suppose that $m \in M$ and that $1 \otimes_{\mathscr{B}} m=0$. Then there exist $b_{i} \in \mathscr{B}$, $m_{j} \in M$, and $a_{i j} \in \mathbf{D}(V)^{W}, i=1, \ldots, r, j=1, \ldots, s$, such that

$$
1 \otimes m=\sum_{i j}\left\{a_{i j} b_{i} \otimes m_{j}-a_{i j} \otimes b_{i} m_{j}\right\}
$$

Let $k$ be so large that $\pi^{2 k} a_{i j} \in \mathscr{B}$ for all $i, j$. We have

$$
\pi^{2 k} \otimes m=\sum_{i j}\left\{\pi^{2 k} a_{i j} b_{i} \otimes m_{j}-\pi^{2 k} a_{i j} \otimes b_{i} m_{j}\right\} .
$$

Hence, $\pi^{2 k} \otimes_{\mathscr{B}} m=0$ as an element of $\mathscr{B} \otimes_{\mathscr{B}} M$. This implies that $\pi^{2 k} m=0$. Hence $m=0$.

Let $\mathscr{C}_{W}$ be the full subcategory of all finitely generated $\mathbf{D}(V)^{W}$ modules, $M$, such that if $m \in M$ and $p \in S_{+}(V)^{W}$ then there exists $k$ such that $p^{k} m=0$ (i.e., each $p \in S_{+}(V)^{W}$ acts locally nilpotently). Let $\check{\mathscr{C}}_{W}$ denote the full subcategory of all $M \in \check{\mathscr{M}}$ such that each $p \in S_{+}(V)^{W}$ acts locally nilpotently. We note that if $M \in \check{\mathscr{C}}_{W}$ then $\mathbf{D}(V)^{W} \otimes_{\mathscr{B}} M \in \mathscr{C}_{W}$. We also note that any $\mathscr{B}$-submodule of $\mathscr{P}(V)$ is in $\check{\mathscr{C}}_{W}$.

Theorem 2.6. If $\lambda \in \widehat{W}$ then $V^{\lambda}$ is an irreducible object in $\check{\mathscr{C}}_{W}$.

(i) If $\lambda, \mu \in \widehat{W}$ and if $V^{\lambda}$ is isomorphic with $V^{\mu}$ as $\mathscr{B}$-modules then $\lambda=\mu$.

(ii) If $M \in \check{\mathscr{C}}_{W}$ then $M$ is isomorphic with $\bigoplus_{\lambda \in \widehat{W}} m_{\lambda} V^{\lambda}$.

Proof. We realize $V^{\lambda}$ as above. Then $V^{\lambda} \in \check{\mathscr{C}}_{W}$. Theorem 2.2 implies that $V^{\lambda}$ is irreducible as a $\mathscr{B}$-module. Suppose that $\lambda, \mu \in \widehat{W}, \lambda \neq \mu$ and that $T$ is a $\mathscr{B}$-module homomorphism from $V^{\lambda}$ to $V^{\mu}$. Let $h \in V^{\lambda}, h \neq 0$, and assume that $h$ is contained in an irreducible $W$-submodule of $\mathscr{P}(V)[\lambda]$ and $T h=u \in V^{\mu}$. As in the proof of Proposition 1.5 there exists $D \in \mathbf{D}(V)^{W}$ such that $D h=h$ and $D u=0$. Let $k$ be such that $\pi^{2 k} D \in \mathscr{B}$. Then $\pi^{2 k} u=T\left(\pi^{2 k} D h\right)=\pi^{2 k} D T h=\pi^{2 k} D u=0$. Thus $u=0$. Since $V^{\lambda}$ is irreducible as a $\mathscr{B}$-module, this implies that $T=0$. This implies (i).

We now prove (ii). We have observed that $N=\mathbf{D}(V)^{W} \otimes_{\mathscr{B}} M \in \mathscr{C}_{W}$. Theorem 1.7 implies that, as a $\mathbf{D}(V)^{W}$-module, $N=\bigoplus_{\lambda \in \widehat{W}} n_{\lambda} V^{\lambda}$. Lemma 2.3 implies that the map $m \mapsto 1 \otimes_{\mathscr{B}} m$ defines an injective $\mathscr{B}$-module homomorphism of $M$ into $N$. This implies (ii).

Let $h_{1}, \ldots, h_{n}$ be an orthonormal basis of $V$, and let $h_{1}^{*}, \ldots, h_{n}^{*}$ be the dual basis. As in Appendix 1 we have an automorphism, $\phi$, of $\mathbf{D}(V)$ such that $\phi\left(h_{j}\right)=i h_{j}^{*}$ and $\phi\left(h_{j}^{*}\right)=i h_{j}$. Then $\phi\left(\mathbf{D}(V)^{W}\right)=\mathbf{D}(V)^{W}$ and $\phi(\mathscr{B})=\mathscr{B}$. Let $\mathscr{C}_{W}^{\prime}$ be the full subcategory of all finitely generated $\mathbf{D}(V)^{W}$-modules, $M$, such that if $p \in \mathscr{P}_{+}(V)^{W}$ then $p$ acts locally nilpotently on $M$. Let $\check{\mathscr{C}}_{W}^{\prime}$ denote the full subcategory of all finitely generated $\mathscr{B}$-modules, $M$, such that if $p \in \mathscr{P}_{+}(V)^{W}$ then $p$ acts locally nilpotently on $M$ and $\sigma\left(\pi^{2}\right)$ acts injectively 
on $M$. If $M \in \mathscr{C}_{W}$ (resp. $M \in \check{\mathscr{C}}_{W}$ ), we define $\widetilde{M}$ to be the $\mathbf{D}(V)^{W}$-module (resp. $\mathscr{B}$-module) with space $M$ and action $D \cdot m=\sigma(D) m$. We define a functor from $\check{\mathscr{C}}_{W}$ to $\check{\mathscr{C}}_{W}^{\prime}$ by $M \rightarrow \widetilde{M}$ and if $T$ is in $\operatorname{Hom}_{\check{\mathscr{C}}_{W}}(M, N)$ then $\widetilde{T}$ is $T$ as a linear map. Then $M \rightarrow \widetilde{M}$ defines an equivalence of categories between $\check{\mathscr{C}}_{W}$ and $\check{\mathscr{C}}_{W}^{\prime}$. We therefore have

Theorem 2.7. If $\lambda \in \widehat{W}$ then $\widetilde{V}^{\lambda}$ is an irreducible object in $\check{\mathscr{C}}_{W}^{\prime}$.

(i) If $\lambda, \mu \in \widehat{W}$ and if $\widetilde{V}^{\lambda}$ is isomorphic with $\widetilde{V}^{\mu}$ as $\mathscr{B}$-modules then $\lambda=\mu$.

(ii) If $M \in \check{\mathscr{C}}_{W}^{\prime}$ then $M$ is isomorphic with $\bigoplus_{\lambda \in \widehat{W}} m_{\lambda} \widetilde{V}^{\lambda}$.

\section{INVARIANT POLYNOMIAL DIFFERENTIAL OPERATORS ON A REDUCTIVE LIE ALGEBRA}

Let $\mathfrak{g}$ be a reductive Lie algebra over $\mathrm{C}$. Let $G$ denote the group of automorphisms of $\mathfrak{g}$ generated by those of the form $e^{a d x}, x \in \mathfrak{g}$. We fix a nondegenerate symmetric bilinear form, $B$, on $\mathfrak{g}$ such that $B([x, y], z)=-B(y,[x, z])$ for $x, y, z \in \mathfrak{g}$. We assume (as we may) that there exists a real form $\mathfrak{g}_{u}$ of $\mathfrak{g}$ such that $B_{\mid \mathfrak{g}_{u}}$ is negative definite. Let $\mathfrak{h}$ be a Cartan subalgebra of $\mathfrak{g}$, and let $\Phi=\Phi(\mathfrak{g}, \mathfrak{h})$ be the root system of $\mathfrak{g}$ relative to $\mathfrak{h}$. Let $W$ denote the Weyl group of $\mathfrak{g}$ with respect to $\mathfrak{h}$. We recall Harish-Chandra's construction of a "radial component" map from differential operators on $\mathfrak{g}$ to differential operators on $\mathfrak{h}^{\prime}=\{h \in \mathfrak{h} \mid \alpha(h) \neq 0, \alpha \in \Phi\}$. For details in the construction the reader could refer to [RRGI,7.A.2].

Let $V$ be a finite-dimensional vector space over $\mathbf{C}$. Let $U$ be an open subset of $V$. Let $\mathbf{D}_{\text {hol }}(U)$ denote the algebra of all differential operators on $U$ with holomorphic coefficients. That is, if $x_{1}, \ldots, x_{n}$ are linear coordinates on $U$ then $D \in \mathbf{D}_{\text {hol }}(U)$ means that

$$
D=\sum_{|I| \leq k} a_{I}(x) \partial^{I}
$$

with $k$ the order of $D$ and $a_{I}$ holomorphic on $U$ and some $a_{I} \neq 0$ with $|I|=k$. As usual, we identify $S(V)$ with the constant coefficient differential operators on $V$. If $D$ is given as above and if $x \in U$ is fixed then we set $D_{x} \in S(V)$ equal to the constant coefficient differential operator with coefficients $a_{I}(x)$.

If $X \in \mathfrak{g}$ then we define $\tau(X)$ to be the vector field on $\mathfrak{g}$ given by

$$
\tau(X) f(y)=\frac{d}{d t} f\left(e^{-\operatorname{tad} X} y\right)_{t=0} .
$$

Then $\tau$ defines a Lie algebra homomorphism of $\mathfrak{g}$ into $\mathbf{D}(\mathfrak{g})$, and hence $\tau$ has a canonical extension to the universal enveloping algebra of $\mathfrak{g}, U(\mathfrak{g})$. We define a linear map

$$
\Gamma: S(\mathfrak{g}) \otimes U(\mathfrak{g}) \rightarrow \mathbf{D}(\mathfrak{g})
$$

by $\Gamma(p \otimes u)=p \tau(u)$. We set $\Gamma_{x}(p \otimes u)=\Gamma(p \otimes u)_{x}$ for $x \in \mathfrak{g}$. Let symm denote the usual symmetrization map of $S(\mathfrak{g})$ onto $U(\mathfrak{g})$. Let $Z=\{x \in \mathfrak{g} \mid B(x, \mathfrak{h})=$ 
$0\}=[\mathfrak{h}, \mathfrak{g}]=\sum_{\alpha \in \Phi} \mathfrak{g}_{\alpha}$ where $\mathfrak{g}_{\alpha}$ is the root space corresponding to $\alpha$. Let $\mathscr{Z}=\operatorname{symm} S(Z)$. Set

$$
(S(\mathfrak{h}) \otimes \mathscr{Z})^{k}=\sum_{i+j \leq k} S_{i}(\mathfrak{h}) \otimes \operatorname{symm}\left(S_{j}(Z)\right) .
$$

If $h \in \mathfrak{h}$ then $\Gamma_{h}:(S(\mathfrak{h}) \otimes \mathscr{Z})^{k} \rightarrow \sum_{j \leq k} S_{j}(\mathfrak{g})=S^{k}(\mathfrak{g})$. Also,

$$
h \mapsto \Gamma_{\left.h\right|_{(S(\mathfrak{h}) \otimes \mathscr{Z})^{k}}}=\Lambda_{h, k}
$$

is a polynomial map from $\mathfrak{h}$ to $\operatorname{Hom}_{\mathbf{C}}\left((S(\mathfrak{h}) \otimes \mathscr{Z})^{k}, S^{k}(\mathfrak{g})\right)$. Furthermore, if $h \in \mathfrak{h}^{\prime}$ then $\Lambda_{h, k}$ is invertible and there exists a natural number $m_{k}$ such that $h \mapsto \pi(h)^{m_{k}}\left(\Lambda_{h, k}\right)^{-1}$ is a polynomial map. Here we fix $\Phi^{+}$, a system of positive roots for $\Phi$, and set $\pi=\prod_{\alpha \in \Phi^{+}} \alpha$.

Let $\mathbf{D}_{\text {hol }, k}(\mathfrak{g})$ denote the space of elements of $\mathbf{D}_{\text {hol }}(\mathfrak{g})$ of order at most $k$. Let $\varepsilon$ denote the homomorphism of $U(\mathfrak{g})$ to $\mathbf{C}$ with kernel $U(\mathfrak{g}) \mathfrak{g}$. Then following Harish-Chandra we define for, $D \in \mathbf{D}_{\text {hol }, k}(\mathfrak{g}), r(D) \in \mathbf{D}_{\text {hol, } k}\left(\mathfrak{h}^{\prime}\right)$ to be the element defined by

$$
r(D)_{h}=(I \otimes \varepsilon) \Lambda_{h, k}^{-1}\left(D_{h}\right) .
$$

Notice that there exists $m_{k} \in \mathbf{N}$ such that $\pi^{m_{k}} r(D) \in \mathbf{D}(\mathfrak{h})$. The definition of $r$ implies that if $D \in \mathbf{D}(\mathfrak{g})^{G}$ then $r(D) \in \mathbf{D}_{\text {hol }}\left(\mathfrak{h}^{\prime}\right)^{W}$. We now recall some results of Harish-Chandra related to this construction.

(1) If $D_{1}, D_{2} \in \mathbf{D}_{\text {hol }}(\mathfrak{g})^{G}$ then $r\left(D_{1} D_{2}\right)=r\left(D_{1}\right) r\left(D_{2}\right)$.

Since $\mathfrak{g}=\mathfrak{h} \oplus Z$, we have a direct sum decomposition $\mathfrak{g}^{*}=\mathfrak{h}^{*} \oplus Z^{*}$. If $p \in$ $S(\mathfrak{g})$ then we look upon $p^{*}$ as an element of $\mathscr{P}\left(\mathfrak{g}^{*}\right)$. We set $\operatorname{Res}_{\mathfrak{g} / \mathfrak{h}}(p)=p_{\mid h^{*}}$.

(2) If $f \in \mathscr{P}(\mathfrak{g})^{G}$ then $r(f)=f_{\mid \mathfrak{h}}$. If $p \in S(\mathfrak{g})^{G}$ then $r(p)=\pi^{-1}\left(\operatorname{Res}_{\mathfrak{g} / \mathfrak{h}} p\right) \pi$.

Let $\mathscr{I}=\left\{D \in \mathbf{D}(\mathfrak{g})^{G} \mid r(D)=0\right\}$.

(3) $\mathscr{I}=\left\{D \in \mathbf{D}(\mathfrak{g})^{G} \mid D \mathscr{P}(\mathfrak{g})^{G}=0\right\}$.

In the next section we will give a more direct characterization of $\mathscr{J}$. Let $\mathscr{A}$ be the subalgebra of $\mathbf{D}(\mathfrak{g})^{G}$ generated by $\mathscr{P}(\mathfrak{g})^{G}$ and $S(\mathfrak{g})^{G}$.

Theorem 3.1. Assume that $(W, \mathfrak{h})$ is good (in the sense of $\S 2$ ). There exists a homomorphism, $\delta$, from $\mathbf{D}(\mathfrak{g})^{G}$ to $\mathbf{D}(\mathfrak{h})^{W}$ with $\operatorname{ker} \delta=\mathscr{J}$ and $\delta(f)=f_{\mid \mathfrak{h}}$ for $f \in \mathscr{P}(\mathfrak{g})^{G} . \delta(p)=\operatorname{Res}_{\mathfrak{g} / \mathfrak{h}}(p)$ for $p \in S(\mathfrak{g})^{G}$. Furthermore the following sequence of algebra homomorphisms is exact:

$$
0 \rightarrow \mathscr{I} \rightarrow \mathbf{D}(\mathfrak{g})^{G} \stackrel{\delta}{\longrightarrow} \mathbf{D}(\mathfrak{h})^{W} \rightarrow 0 .
$$

Proof. (1) and (2) above imply that if we set $\delta(D)=\pi \circ r(D) \circ \pi^{-1}$ on $\mathscr{A}$ then $\delta$ is an algebra homomorphism onto the subalgebra of $\mathbf{D}(\mathfrak{h})$ generated by $\mathscr{P}(\mathfrak{h})^{W}$ and $S(\mathfrak{h})^{W}$. Since $(\boldsymbol{W}, \mathfrak{h})$ is good, Proposition 2.1 implies that $\delta(\mathscr{A})=\mathbf{D}(\mathfrak{h})^{W}$. That $\delta$ extends to a homomorphism of $\mathbf{D}(\mathfrak{g})^{G}$ to $\mathbf{D}(\mathfrak{h})^{W}$ is due to Harish-Chandra [H3].

Corollary 3.2. If $\mathfrak{g}$ has no simple ideals of type $E$ then the conclusion of Theorem 3.1 is true for $\mathfrak{g}$. 
This is a direct consequence of Corollary 2.2.

We will say that $\mathfrak{g}$ is "nice" if the conclusion of Theorem 3.1 is true for $\mathfrak{g}$. Thus we know that $\mathfrak{g}$ is nice if it contains no simple ideals of type $E$.

\section{A CLOSER EXAMINATION OF THE IDEAL $\mathscr{I}$}

We retain the notation of the previous section. If $X \in \mathfrak{g}$ then we write

$$
\operatorname{det}(\operatorname{ad} X+t I)=\sum_{j} t^{j} d_{j}(X) .
$$

If $\operatorname{dim} \mathfrak{h}=l$ then $d_{j}=0$ for $j<l$ and $d_{l}$ is a nonzero polynomial function on $\mathfrak{g}$. The object of this section is to prove

Lemma 4.1. $D \in \mathscr{I}$ if and only if there exists $k \in \mathbf{N}$ such that $d_{l}^{k} D \in$ $(\mathbf{D}(\mathfrak{g}) \tau(\mathfrak{g})) \cap \mathbf{D}(\mathfrak{g})^{G}$.

We note that if $D \in \mathbf{D}(\mathfrak{g})^{G}$ and there exists $k \in \mathbf{N}$ such that $d_{l}^{k} D \in \mathbf{D}(\mathfrak{g}) \tau(\mathfrak{g})$ then $d_{l}^{k} D \mathscr{P}(\mathfrak{g})^{G}=0$. Thus $D \mathscr{P}(\mathfrak{g})^{G}=0$. So $D \in \mathscr{I}$. Before we begin the preparations for the proof of the converse assertion we first give a conjectural sharpening of the lemma.

Question. If $\mathscr{I}=(\mathbf{D}(\mathfrak{g}) \tau(\mathfrak{g})) \cap \mathbf{D}(\mathfrak{g})^{G}$ ?

We note that the answer to this question would be yes if one could show that the ideal in $\mathscr{P}(\mathfrak{g} \times \mathfrak{g})$ generated by the polynomials $X, Y \mapsto B(Z,[X, Y])$, $Z \in \mathfrak{g}$, is a prime ideal (it is a well-known result of Richardson that the corresponding affine algebraic set is irreducible).

We will now develop some material necessary for our proof of Lemma 4.1. Let $\varphi_{1}, \ldots, \varphi_{l}(l=\operatorname{dim} \mathfrak{h})$ be algebraically independent generators of $\mathscr{P}(\mathfrak{g})^{G}$ such that each $\varphi_{j}$ takes real values on $\mathfrak{g}_{u}$. Let, for $X \in \mathfrak{g}, \Psi_{i}(X) \in \mathfrak{g}$ be defined by $B\left(\Psi_{i}(X), Y\right)=d \varphi_{i_{X}}(Y)$. Then $\Psi_{i}$ is a polynomial mapping of $\mathfrak{g}$ to $\mathfrak{g}$ and

(1) $\left[X, \Psi_{i}(X)\right]=\left[\Psi_{i}(X), \Psi_{j}(X)\right]=0$ for all $i, j \leq l$.

This is standard. Indeed, since $\varphi_{j} \in \mathscr{P}(\mathfrak{g})^{G}$, it is easily seen that $\left[X, \Psi_{j}(X)\right]$ $=0$ for all $X \in \mathfrak{g}$. If $X \in \mathfrak{g}^{\prime}=\left\{Y \in \mathfrak{g} \mid d_{l}(Y) \neq 0\right\}$ then $C_{\mathfrak{g}}(X)=\{Y \in$ $\mathfrak{g} \mid[X, Y]=0\}$ is a Cartan subalgebra. Thus (1) is true for $X \in \mathfrak{g}^{\prime}$. Since $\Psi_{j}$ is a polynomial map, (1) is true for all $X \in \mathfrak{g}$.

(2) If $X \in \mathfrak{g}^{\prime}$ then $\Psi_{1}(X), \ldots, \Psi_{l}(X)$ is a basis of $C_{\mathfrak{g}}(X)$.

Indeed, if $X \in \mathfrak{g}^{\prime}$, we may choose $\mathfrak{h}=C_{\mathfrak{g}}(X)$. Let $x_{1}, \ldots, x_{l}$ be linear coordinates on $\mathfrak{h}$. If $i$ is the canonical injection of $\mathfrak{h}$ into $\mathfrak{g}$ then

$$
i^{*}\left(d \varphi_{1} \wedge \cdots \wedge d \varphi_{l}\right)=c \pi d x_{1} \wedge \cdots \wedge d x_{l}
$$

with $c \neq 0$. This implies that $\Psi_{1}(X), \ldots, \Psi_{l}(X)$ are linearly independent. (2) now follows from (1).

One can show that if $X \in \mathfrak{g}$ then $\operatorname{dim} C_{\mathfrak{g}}(X)=l$ if and only if $\Psi_{1}(X), \ldots$, $\Psi_{l}(X)$ are linearly independent. This was first observed by Kostant. 
(3) If $X \in \mathfrak{g}^{\prime}$ then $C_{\mathfrak{g}}(X)^{\perp}=[X, \mathfrak{g}]$.

Indeed, if $C_{\mathfrak{g}}(X)=\mathfrak{h}$ then $C_{\mathfrak{g}}(X)^{\perp}=\sum_{\alpha \in \Phi} \mathfrak{g}_{\alpha}$. Since $[\mathfrak{h}, \mathfrak{h}]=0$ and $\mathfrak{a}(X) \neq 0$ for all $\alpha \in \mathfrak{h},(3)$ follows.

We regard $\mathfrak{g}^{\prime}$ as the affine variety $V=\left\{(x, t) \in \mathfrak{g} \times C \mid d_{l}(x) t=1\right\}$.

If $X \in \mathfrak{g}$ then $\left[X, X_{i}\right] \in C_{\mathfrak{g}}(X)^{\perp}$. Fix $X_{o} \in \mathfrak{g}^{\prime}$, and let $X_{1}, \ldots, X_{n}$ be a basis of $C_{\mathfrak{g}}\left(X_{o}\right)^{\perp}$. Then $\left[X_{o}, X_{1}\right], \ldots,\left[X_{o}, X_{n}\right]$ is also a basis of $C_{\mathfrak{g}}\left(X_{o}\right)^{\perp}$. We set

$$
U_{X_{o}}=\left\{X \in \mathfrak{g}^{\prime} \mid\left[X, X_{1}\right] \wedge \cdots \wedge\left[X, X_{n}\right] \neq 0\right\} .
$$

Then $U_{X_{o}}$ is Zariski open in $V$. Put, for $X \in \mathfrak{g}, u_{j}(X)=\left[X, X_{j}\right]$. Then $u_{j}$ is a polynomial map of $\mathfrak{g}$ to $\mathfrak{g}$ and $u_{j}(X) \in C_{\mathfrak{g}}(X)^{\perp}$. Furthermore, if $X \in U_{X_{o}}$ then $u_{1}(X), \ldots, u_{n}(X)$ is a basis if $C_{\mathfrak{g}}(X)^{\perp}$.

We now take a closer look at the maps $\Lambda_{h, k}$. If $X \in \mathfrak{g}^{\prime}$ then we define

$$
\Lambda_{X, k}:\left(S\left(C_{\mathfrak{g}}(X)\right) \otimes \operatorname{symm}\left(S\left(C_{\mathfrak{g}}(X)^{\perp}\right)\right)^{k} \rightarrow S^{k}(\mathfrak{g})\right.
$$

to be the restriction of $\Gamma_{X}$. If $p \in S(\mathfrak{g}), x \in U(\mathfrak{g})$, and $g \in G$ then we set

$$
g(p \otimes x)=g p \otimes \operatorname{Ad}(g) x .
$$

We have

$$
\Gamma_{\operatorname{Ad}(g) X}(g u)=g \Gamma_{X}(u) .
$$

If $D \in \mathbf{D}_{\text {hol }, k}(\mathfrak{g})^{G}$ and if $g \in G$ then $g \cdot D_{\operatorname{Ad}(g) X}=D_{X}$. If $X \in U_{X_{o}}$ then there exists $g \in G$ such that $\operatorname{Ad}(g) C_{\mathfrak{g}}(X)=\mathfrak{h}$. Thus if $D \in \mathbf{D}_{\text {hol }, k}(\mathfrak{g})^{G}$ and if $r(D)=0$ then

$$
(I \otimes \varepsilon)\left(\Lambda_{X, k}^{-1}\left(D_{X}\right)\right)=0
$$

for all $X \in \mathfrak{g}^{\prime}$. We set $\Psi(X)^{I}=\Psi_{1}(X)^{i_{1}} \ldots \Psi_{l}(X)^{i_{l}}$ and $u(X)^{J}=u_{1}(X)^{j_{1}} \ldots$ $u_{n}(X)^{j_{n}}$. Let $Y_{1}, \ldots, Y_{m}$ be a basis of $\mathfrak{g}(m=n+l)$, and set $Y^{N}=Y_{1}^{n_{1}} \cdots Y_{m}^{n_{m}}$ in $S(\mathfrak{g})$. Then if $|I|+|J| \leq k$, we have

$$
\Lambda_{X, k}\left(\Psi(X)^{I} \otimes \operatorname{symm}\left(u(X)^{J}\right)\right)=\sum_{|N| \leq k} a_{N, I, J}(X) Y^{N}
$$

with $a_{N, I, J}$ a polynomial in $X$. Let $Z_{1}, \ldots, Z_{m}$ be a basis of $\mathfrak{g}$, and set $Z^{N}=Z_{1}^{n_{1}} \cdots Z_{m}^{n_{m}}$ in $U(\mathfrak{g})$. Then these observations imply that if $D \in \mathbf{D}^{k}(\mathfrak{g})^{G}$ and if $r(D)=0$ then

$$
\Lambda_{X, k}^{-1}\left(D_{X}\right)=\sum_{|P|+|Q| \leq k} b_{P, Q}(X) Y^{P} \otimes Z^{Q}
$$

with $b_{P, Q}$ a rational function on $X$ defined for $X \in U_{X_{o}}$ and $b_{P, Q}=0$ if $Q=0$. Since $X_{o} \in V$ is arbitrary, the expression (1) is valid for all $X \in V$ with $b_{P, Q}$ a regular function on $V$. Thus

$$
D_{X}=\sum_{\substack{|P|+|Q| \leq k \\|Q|>0}} b_{P, Q}(X) Y^{P} \tau\left(Z^{Q}\right)
$$


with $b_{P, Q}$ a regular function on $V$. The ring of regular functions on $V$ is $\mathscr{P}(\mathfrak{g})\left[d_{l}^{-1}\right]$. Let $k \in \mathbf{N}$ be such that $d_{l}^{k} b_{P, Q} \in \mathscr{P}(\mathfrak{g})$. Then $d_{l}^{k} D \in \mathbf{D}(\mathfrak{g}) \tau(\mathfrak{g})$. Hence

$$
d_{l}^{k} D \in \mathbf{D}(\mathfrak{g}) \tau(\mathfrak{g}) \cap \mathbf{D}(\mathfrak{g})^{G}
$$

This completes the proof of the lemma.

\section{A THEOREM OF HARISH-ChANDRA}

Let $\mathfrak{g}_{O}$ be a reductive Lie algebra over $\mathbf{R}$. Let $\mathfrak{g}$ denote the complexification of $\mathfrak{g}_{o}$. We assume throughout this section that $\mathfrak{g}$ is nice (see the end of $\S 3$ ). We choose $B$ such that there is a real form $\mathfrak{g}_{u}$ of $\mathfrak{g}$ such that $B_{\mid \mathfrak{g}_{u}}$ is negative definite and such that $B\left(\mathfrak{g}_{o}, \mathfrak{g}_{o}\right) \subset \mathbf{R}$. This clearly can be done. We denote by $G_{u}$ the subgroup of $G$ generated by $\left\{e^{a d X} \mid X \in \mathfrak{g}_{u}\right\}$. Let $\mathscr{S}\left(\mathfrak{g}_{o}\right)$ be the usual Schwartz space of $\mathfrak{g}_{o}$ with the usual Fréchet space topology. If $f \in \mathscr{S}\left(\mathfrak{g}_{o}\right)$ then we use $B$ to define $\mathscr{F}_{B}(f)=\mathscr{F}(f)=\hat{f} \in \mathscr{S}\left(\mathfrak{g}_{o}\right)$ as in Appendix 1. Let $\phi$ be defined as in Appendix 1 for an orthonormal basis of $\mathfrak{g}$ with respect to $B$. We will also write $\phi(D)=\widehat{D}$. We note (as in Appendix 1) that $\mathscr{F}(D f)=\widehat{D} \mathscr{F}(f)$ for $D \in \mathbf{D}(\mathfrak{g})$ and $f \in \mathscr{S}\left(\mathfrak{g}_{o}\right)$.

Let $\mathscr{I}$ be as in the previous sections.

Lemma 5.1. If $D \in \mathscr{I}$ then $\widehat{D} \in \mathscr{I}$.

Proof. We first observe that if $\mathscr{S}\left(\mathfrak{g}_{u}\right)^{G_{u}}=\left\{f \in \mathscr{S}\left(\mathfrak{g}_{u}\right) \mid f(\operatorname{Ad}(u) x)=f(x), u \in\right.$ $\left.G_{u}, x \in \mathfrak{g}_{u}\right\}$ then

(1) If $D \in \mathbf{D}(\mathfrak{g})^{G}$ then $D \in \mathscr{I}$ if and only if $D \mathscr{S}\left(\mathfrak{g}_{u}\right)^{G_{\bar{u}}}=0$.

Indeed, Lemma 4.1 implies that $D \mathscr{S}\left(\mathfrak{g}_{u}\right)^{G_{u}}=0$ if $D \in \mathscr{I}$. If $D \mathscr{S}\left(\mathfrak{g}_{u}\right)^{G_{u}}=0$ and if $f \in \mathscr{P}(\mathfrak{g})^{G}, x \in \mathfrak{g}_{u}$ let $\varphi \in C_{c}^{\infty}\left(\mathfrak{g}_{u}\right) \cap \mathscr{S}\left(\mathfrak{g}_{u}\right)^{G_{u}}$ be such that $\varphi$ is identically equal to 1 in a neighborhood of $x$ in $\mathfrak{g}_{u}$. Then $0=(D(\varphi f))(x)=$ $D f(x)$. Thus $D \in \mathscr{I}((3)$ in $\S 3)$.

Now if $D \in \mathscr{I}$ then $\mathscr{F}(D f)=0$ for all $f \in \mathscr{S}\left(\mathfrak{g}_{u}\right)^{G_{u}}$. Thus $\widehat{D} \hat{f}=0$ for all $f \in \mathscr{S}\left(\mathfrak{g}_{u}\right)^{G_{u}}$. Since $\mathscr{F}$ is a linear bijection of $\mathscr{S}\left(\mathfrak{g}_{u}\right)^{G_{u}}$ onto itself, we see that $\widehat{D} \mathscr{S}\left(\mathfrak{g}_{u}\right)^{G_{u}}=0$. Thus (1) implies that $\widehat{D} \in \mathscr{I}$.

Lemma 5.2. $D \in \mathscr{I}$ if and only if there exists $k \in \mathbf{N}$ such that

$$
\phi\left(d_{l}^{k}\right) D \in(\mathbf{D}(\mathfrak{g}) \tau(\mathfrak{g})) \cap \mathbf{D}(\mathfrak{g})^{G} \text {. }
$$

Proof. We note that if $X \in \mathfrak{g}$ then $(\tau(X) \hat{)}=-\tau(X)$. Thus

$$
\left((\mathbf{D}(\mathfrak{g}) \tau(\mathfrak{g})) \cap \mathbf{D}(\mathfrak{g})^{G}\right)^{\widehat{y}}=(\mathbf{D}(\mathfrak{g}) \tau(\mathfrak{g})) \cap \mathbf{D}(\mathfrak{g})^{G} .
$$

Let $D \in \mathscr{I}$, and let $D_{1} \in \mathscr{I}$ be such that $\widehat{D}_{1}=D$. Let $k \in \mathbf{N}$ be such that

$$
d_{l}^{k} D_{1} \in(\mathbf{D}(\mathfrak{g}) \tau(\mathfrak{g})) \cap \mathbf{D}(\mathfrak{g})^{G}
$$

Then

$$
\hat{d}_{l}^{k} D=\hat{d}_{l}^{k} \widehat{D}_{1}=\left(d_{l}^{k} D_{1} \hat{)} \in(\mathbf{D}(\mathfrak{g}) \tau(\mathfrak{g})) \cap \mathbf{D}(\mathfrak{g})^{G} .\right.
$$

The converse is proved in the same way. 
Let $G_{o}$ be the subgroup of $G$ generated by $\left\{e^{a d x} \mid x \in \mathfrak{g}_{o}\right\}$. If $\Omega$ is a subset of $\mathfrak{g}_{o}$, it will be called invariant if $g \Omega \subset \Omega$ for all $g \in G_{o}$. If $\Omega$ is an open invariant subset of $\mathfrak{g}_{o}$ then $\Omega$ will be called completely invariant if, for each $X \in \Omega, X_{s} \in \Omega$. Here if $X \in \mathfrak{g}_{o}$ then $X_{s}, X_{n} \in \mathfrak{g}_{o}$ are uniquely determined by the conditions that $\left[X_{s}, X_{n}\right]=0$, ad $X_{s}$ is diagonalizable on $\mathfrak{g}, X_{n} \in\left[\mathfrak{g}_{o}, \mathfrak{g}_{o}\right]$, and $\operatorname{ad}\left(X_{n}\right)$ is nilpotent. Set $\mathscr{N}=\left\{X \in \mathfrak{g}_{o} \mid X=X_{n}\right\}$. Then $\mathscr{N}=\left\{X \in \mathfrak{g}_{o} \mid f(X)=f(0), f \in \mathscr{P}(\mathfrak{g})^{G}\right\}$ (cf. [RRGI, 8.A.4.2]).

If $\Omega$ is an invariant open subset of $\mathfrak{g}_{o}$ and if $T \in \mathscr{D}^{\prime}(\Omega)$ (distributions on $\Omega)$ then we define $g T(f)=T\left(g^{-1} f\right)\left(g f(x)=f\left(g^{-1} x\right)\right)$. We set $\mathscr{D}^{\prime}(\Omega)^{G_{o}}=$ $\left\{T \in \mathscr{D}^{\prime}(\Omega) \mid g T=T, g \in G_{o}\right\}$ We now recall a basic theorem of HarishChandra ([H2], cf. [RRG1, Theorem 8.3.5].

Theorem 5.3. Let $\Omega$ be an open completely invariant subset of $\mathfrak{g}_{o}$, and assume that $T \in \mathscr{D}^{\prime}(\Omega)^{G_{o}}$ is such that $\operatorname{dim} S(\mathfrak{g})^{G} T<\infty$. If $T_{\mid \Omega \cap \mathfrak{g}^{\prime}}=0$ then $T=0$.

We will now give a new proof of the following theorem of Harish-Chandra. We note that an affirmative answer to the question in the last section would directly imply the theorem. If $\mathfrak{g}$ is nice our proof is independent of Theorem 5.3 and for such $\mathfrak{g}_{o}$ we show how the next result (combined with our theory) implies Theorem 5.3.

Theorem 5.4. If $\Omega$ is an open, nonempty, completely invariant subset of $\mathfrak{g}_{o}$ then

$$
\mathscr{I}=\left\{D \in \mathbf{D}(\mathfrak{g})^{G} \mid D \mathscr{D}^{\prime}(\Omega)^{G_{o}}=0\right\} .
$$

The first part of our proof follows the same line of the original argument of Harish-Chandra (we will refer to the exposition in Varadarajan [Var]). The proof is by induction on $\operatorname{dim} \mathfrak{g}$. If $\operatorname{dim} \mathfrak{g}<3$ then $\mathscr{I}=\{0\}$, so the result is clear. Assume the result for $2 \leq \operatorname{dim} \mathfrak{g}<r$. We look at the case $\operatorname{dim} \mathfrak{g}=r$. We refer to [Var, pp. 149-150] for the reduction of the inductive step to the case when $\mathfrak{g}$ is semisimple and if $D \in \mathscr{I}, \operatorname{supp} D T \subset \mathscr{N}$. Thus Lemma 2 in Appendix 3 implies that $D T$ extends to a tempered distribution on $\mathfrak{g}_{o}$. Since $\operatorname{supp} D T \subset \mathscr{N}$, if $f \in \mathscr{P}_{+}(\mathfrak{g})^{G}$ then there exists $k \in \mathbf{N}$ such that $f^{k} D T=0$. Applying the Fourier transform, this implies that if $p \in S_{+}(\mathfrak{g})^{G}$ then there exists $k \in \mathbf{N}$ such that $p^{k}(D T \hat{)}=0$.

We now assume that $\mathfrak{g}$ is nice. Let $D_{1} \in \mathscr{I}$; then

$$
\operatorname{dim} S(\mathfrak{g})^{G} D_{1}(D T)<\infty .
$$

Since $\operatorname{supp} D_{1}(D T) \subset \mathscr{N}$, we also have

$$
\operatorname{dim} \mathscr{P}(\mathfrak{g})^{G} D_{1}(D T)<\infty .
$$

We consider the operators $e=\frac{1}{2} B(\ldots, \ldots), f=\phi(e), \quad h=[e, f]=$ $\sum x_{i} \partial / \partial x_{i}+m / 2$ (here $x_{i}$ is any system of linear coordinates for $\left.\mathfrak{g}_{o}\right)$. Then $\{e, f, h\}$ span a Lie algebra, $u$, over $\mathbf{C}$ isomorphic with $\mathfrak{s l}(2, \mathbf{C})$. The above implies that $\operatorname{dim} U(\mathfrak{u}) D_{1}(D T)<\infty$. Thus Lemma 8.3.7 in [RRGI] implies that $D_{1}\left(D T \hat{)}=0\right.$. Since $\widehat{\mathscr{I}}=\mathscr{I}$, this implies that $\mathscr{I} D T=0$. Hence $\mathbf{D}(\mathfrak{g})^{G} D T$ 
pushes down (via $\delta$ ) to a $\mathbf{D}(\mathfrak{h})^{W}$-module, $N$, with the property that if $n \in N$ then there exists $k \in \mathbf{N}$ such that $\mathscr{P}_{k}(\mathfrak{h})^{W} n=0$. On the other hand, there exists $j \in \mathbf{N}$ such that $\phi\left(d_{l}\right)^{j} D \in \mathbf{D}(\mathfrak{g}) \tau(\mathfrak{g}) \cap \mathbf{D}(\mathfrak{g})^{G}$. Thus $\phi\left(d_{l}\right)^{j}(D T)=0$. Hence if $n_{o}$ is the element of $N$ corresponding to $D T$ then $\delta\left(\phi\left(d_{l}\right)^{j}\right) n_{o}=0$. Corollary 1.4 now implies that $n_{o}=0$. Thus $D T=0$. This completes the proof if $\mathfrak{g}$ is nice.

We now give the general argument (which uses Theorem 5.3). Lemma $5.2 \mathrm{im}-$ plies that there exists $k \in \mathbf{N}$ such that $\phi\left(d_{l}\right)^{k} D \in \mathbf{D}(\mathfrak{g})^{G} \tau(\mathfrak{g})$. Thus $\phi\left(d_{l}\right)^{k} D T=$ 0 . Hence $d_{l}^{k} \mathscr{F}(D T)=0$. Set $S=\mathscr{F}(D T)$. Then the observations at the beginning of the proof imply that if $p \in S_{+}(\mathfrak{g})^{G}$ then there exists $r$ such that $p^{r} S=0$. Since $d_{l}^{k} S=0, S_{\mid \Omega \cap g^{\prime}}=0$. Theorem 5.3 implies that $S=0$. Hence $D T=0$, as was to be proved.

We note that the above result implies that if $\Omega$ is an open completely invariant subset of $\mathfrak{g}_{o}$ then the $\mathbf{D}(\mathfrak{g})^{G}$-module $\mathscr{D}^{\prime}(\Omega)^{G_{o}}$ pushes down, via $\delta$, to a $\mathbf{D}(\mathfrak{h})^{W}$-module if $\mathfrak{g}$ is nice. In general it pushes down to a $\mathscr{B}$-module.

We now show how one can use Theorem 5.4 to prove Theorem 5.3 in the case when $\mathfrak{g}$ is nice. So assume that $\mathfrak{g}$ is nice. Set $\mathfrak{g}_{o}^{\prime}=\mathfrak{g}_{o} \cap \mathfrak{g}^{\prime}$. Assume that $\Omega$ is open and completely invariant in $\mathfrak{g}_{o}$ and that $T_{\mid \Omega \cap \mathfrak{g}_{o}^{\prime}}=0$. We show that $T=0$.

Let $U_{j}$ be an increasing sequence of open subsets of $\Omega$ such that $\Omega$ is the union of the $U_{j}$ and that $\bar{U}_{j}$ is compact and contained in $\Omega$. Then $T_{\mid U_{j}}$ is of finite order with $\operatorname{supp} T_{\mid U_{j}} \subset U_{j} \cap\left(\mathfrak{g}_{o}-\mathfrak{g}_{o}^{\prime}\right)$. Hence, for each $j$ there exists $k_{j}$ such that $d_{l}^{k_{j}} T_{\mid U_{j}}=0, k_{1} \leq k_{2} \leq \cdots$. We consider $\mathbf{D}(\mathfrak{g})^{G} T$ to be the $\mathbf{D}(\mathfrak{h})^{W}$-module, $M$, via $\delta$. Let $m \in M$ correspond to $T$. Then we have a decreasing sequence of $\mathbf{D}(\mathfrak{h})^{W}$-submodules, $\mathbf{D}(\mathfrak{h})^{W} \pi^{2 k} m \supset \mathbf{D}(\mathfrak{h})^{W} \pi^{2 k+2} m$. Since $\operatorname{dim} S(\mathfrak{h})^{W} m<\infty$, Proposition 1.3 applies, so $M$ has finite length. Hence there exists $k$ such that

$$
\mathbf{D}(\mathfrak{h})^{W} \pi^{2 k} m=\mathbf{D}(\mathfrak{h})^{W} \pi^{2 p} m
$$

for $p \geq k$. Now this implies that if $p \geq k$ then $d_{l}^{k} T=D_{p} d_{l}^{p} T$ for an appropriate $D_{p} \in \mathbf{D}(\mathfrak{g})^{G}$. We therefore see that if $k_{j} \geq k$ then

$$
d_{l}^{k} T_{\mid U_{j}}=D_{k_{j}} d_{l}^{k_{j}} T_{\mid U_{j}}=0 .
$$

Since the union of the $U_{j}$ is $\Omega$, we have $d_{l}^{k} T=0$. If we revert to $M$, we have $\pi^{2 k} m=0$. Hence Proposition 1.3 implies $m=0$. Thus $T=0$.

Let $\mathscr{D}_{\mathcal{N}}^{\prime}\left(\mathfrak{g}_{o}\right)^{G_{o}}$ denote the space of all $T \in \mathscr{D}^{\prime}\left(\mathfrak{g}_{o}\right)^{G_{o}}$ such that $\operatorname{supp} T \subset$ $\mathscr{N} \cap \mathfrak{g}_{o}$. We now display an implication of the proof of Theorem 5.4.

Proposition 5.5. Let $T \in \mathscr{D}_{\mathcal{N}}^{\prime}\left(\mathfrak{g}_{o}\right)^{G_{o}}$; then, as a $\mathscr{B}$-module, $\mathscr{A} T \in \check{C}_{W}^{\prime}$.

Proof. In the course of the proof we showed that if $f \in \mathscr{P}_{+}(\mathfrak{g})^{G}$ then $f$ acts locally nilpotently on $\mathscr{D}_{\mathcal{N}}^{\prime}\left(\mathfrak{g}_{o}\right)^{G_{o}}$. It also follows (as in the end of the proof of 
Theorem 5.4) that if $T \in \mathscr{D}_{\mathcal{N}}^{\prime}\left(\mathfrak{g}_{o}\right)^{G_{o}}$ and if $\phi\left(d_{l}\right) T=0$ then $T=0$. Since $\delta\left(d_{l}\right)=\pi^{2}$, the result now follows from the definition of $\check{\mathscr{C}}_{W}$.

\section{DisTRIBUTIONS SUPPORTED ON THE NILPOTENT CONE AND WEYL GROUP REPRESENTATIONS}

We will use the notation and conventions of $\S 5$ in this section except that we will take $\mathfrak{g}_{o}$ to be semisimple and $B$ to be the Killing form. Let $\mathscr{N}$ be the cone of all nilpotent $X \in \mathfrak{g}_{o}$ (i.e., $a d X$ is nilpotent). If $X \in \mathscr{N}$ then there exist $Y, H \in \mathfrak{g}_{o}$ such that $[X, Y]=H,[H, X]=2 X,[H, Y]=-2 Y$. Following Kostant we will call $\{X, Y, H\}$ an s-triple containing $X$. Thus $\mathscr{O}_{X}=G_{o} X$ is a cone with $\operatorname{tg} X=g e^{\log (t) a d H / 2} X$ for $t \in \mathbf{R}, t>0$. If $X \in \mathfrak{g}_{o}$ then we set $m(X)=\operatorname{dim} \mathscr{O}_{X} / 2$.

If $S$ is a closed subset of $\mathfrak{g}_{o}$, we denote by $\mathscr{D}_{S}^{\prime}\left(\mathfrak{g}_{o}\right)$ the space of all distributions on $\mathfrak{g}_{o}$ supported on $S$. If $S$ is $G_{o}$-invariant then we set $\mathscr{D}_{S}^{\prime}\left(\mathfrak{g}_{o}\right)^{G_{o}}=$ $\mathscr{D}_{S}^{\prime}\left(\mathfrak{g}_{o}\right) \cap \mathscr{D}^{\prime}\left(\mathfrak{g}_{o}\right)^{G_{o}}$. If $T \in \mathscr{D}_{\mathscr{N}}^{\prime}\left(\mathfrak{g}_{o}\right)^{G_{o}}$ then, as a $\mathscr{B}$-module (via $\delta$ ), $\mathscr{A} T \in \check{\mathscr{C}}_{W}^{\prime}$ (see $\S 2$ and Proposition 5.5).

Theorem 6.1. As a $\mathscr{B}$-module $\mathscr{D}_{\mathscr{N}}^{\prime}\left(\mathfrak{g}_{o}\right)^{G_{o}}$ is equivalent with a direct sum $\bigoplus_{\lambda \in \widehat{W}} m_{\lambda} \widetilde{V}^{\lambda}$ with $m_{\lambda}<\infty$ (notation as in $\S 1.2$ ).

Proof. If we show that $\mathscr{D}_{\mathscr{N}}^{\prime}\left(\mathfrak{g}_{o}\right)^{G_{o}}$ is finitely generated as a $\mathscr{B}$-module then the above observation implies that $\mathscr{D}_{\mathcal{N}}^{\prime}\left(\mathfrak{g}_{o}\right)^{G_{o}} \in \check{\mathscr{C}}_{W}^{\prime}$. The theorem would then follow from Theorem 2.7. We are thus left with the proof of finite generation.

Let $X_{1}, \ldots, X_{m}$ be a basis of $\mathfrak{g}$ such that $B\left(X_{i}, X_{j}\right)=\delta_{i j}$. Let $x_{1}, \ldots, x_{m}$ be the corresponding linear coordinates on $\mathfrak{g}$. Set $r_{\mathfrak{g}}^{2}=\sum_{i} x_{i}^{2}, \Delta_{\mathfrak{g}}=\sum_{i} X_{i}^{2}=$ $\sum_{i} \partial_{i}^{2}$, and $E_{\mathfrak{g}}=\sum_{i} x_{i} \partial_{i}$. Let $H_{1}, \ldots, H_{l}$ be a basis of $\mathfrak{h}$ such that $B\left(H_{i}, H_{j}\right)$ $=\delta_{i j}$, let $y_{i}$ be the corresponding linear coordinates on $\mathfrak{h}$, and set $r_{\mathfrak{h}}^{2}=\sum_{i} y_{i}^{2}$, $\Delta_{\mathfrak{g}}=\sum_{i} H_{i}^{2}=\sum_{i} \partial^{2} / \partial y_{i}^{2}$, and $E_{\mathfrak{h}}=\sum_{i} y_{i} \partial / \partial y_{i}$. Then $\delta\left(r_{\mathfrak{g}}^{2}\right)=r_{\mathfrak{h}}^{2}, \delta\left(\Delta_{\mathfrak{g}}\right)=$ $\Delta_{\mathfrak{h}}$. Thus

$$
\delta\left(E_{\mathfrak{g}}+\frac{m}{2}\right)=\frac{1}{4} \delta\left(\left[\Delta_{\mathfrak{g}}, r_{\mathfrak{g}}^{2}\right]\right)=\frac{1}{4}\left[\Delta_{\mathfrak{h}}, r_{\mathfrak{h}}^{2}\right]=E_{\mathfrak{h}}+\frac{l}{2}
$$

Hence

$$
\delta\left(E_{\mathfrak{g}}\right)=E_{\mathfrak{h}}+\frac{l-m}{2} .
$$

If $T \in \mathscr{D}_{\mathscr{N}}^{\prime}\left(\mathfrak{g}_{o}\right)^{G_{o}}$ then $\mathscr{A} T \in \check{C}_{W}^{\prime}$. Thus, in particular, $E_{\mathfrak{g}}$ acts semisimply on $\mathscr{D}_{\mathscr{N}}^{\prime}\left(\mathfrak{g}_{o}\right)^{G_{o}}$. In light of Theorem 2.7, to prove the finite generation of $\mathscr{D}_{\mathscr{N}}^{\prime}\left(\mathfrak{g}_{o}\right)^{G_{o}}$ it is enough to prove that the dimension of each eigenspace for $E_{\mathfrak{g}}$ in $\mathscr{D}_{\mathscr{N}}^{\prime}\left(\mathfrak{g}_{o}\right)^{G_{o}}$ is finite dimensional. In order to prove this, we set up some notation and a lemma that will be used later in this section. In light of the semisimplicity of $E_{\mathfrak{g}}$ on $\mathscr{D}_{\mathscr{N}}^{\prime}\left(\mathfrak{g}_{o}\right)^{G_{o}}$ the lemma is essentially the same as [BV1, Corollary 3.9]. However, since it is critical to our application, we will include the (not too difficult) proof. 
If $X \in \mathfrak{g}$ then set $\mathfrak{g}^{X}=\operatorname{ker} a d X$. If $X \in \mathfrak{g}_{o}$ then $\mathfrak{g}_{o}^{X}=\mathfrak{g}^{X} \cap \mathfrak{g}_{0}$ is the Lie algebra of $G_{o}^{X}=\left\{g \in G_{o} \mid g X=X\right\}$. Clearly, $m(X)=\frac{1}{2}\left(\operatorname{dim} \mathfrak{g}-\operatorname{dim} \mathfrak{g}^{X}\right)$. Let $\mathscr{O}_{i}=G_{o} X_{i}, i=1, \ldots, r$, be the distinct orbits of $G_{o}$ in $\mathcal{N}$. We assume that $\operatorname{dim} \mathfrak{g}^{X_{i}} \leq \operatorname{dim} \mathfrak{g}^{X_{i+1}}$. Then $\mathscr{O}_{r}=\{0\}$ and $\overline{\mathscr{O}}_{i} \subset \mathscr{O}_{i} \cup\left(\bigcup_{\operatorname{dim} \mathfrak{g}^{X_{j}}>\operatorname{dim} \mathfrak{g}^{X_{i}}} \mathscr{O}_{j}\right)$. Set $\mathscr{N}_{p}=\bigcup_{i \geq p} \mathscr{O}_{i}$ (cf. [RRG1, 8.3]).

The first sentence of the following lemma will complete the proof of the theorem.

Lemma 6.2 (compare [BV1, Corollary 3.9]). $E_{\mathfrak{g}}$ acts semisimply on $\mathscr{D}_{\mathcal{N}_{p}}^{\prime}\left(\mathfrak{g}_{o}\right)^{G_{o}}$ with finite-dimensional eigenspaces and each eigenvalue of $E_{\mathfrak{g}}$ on this space is at most $-\frac{1}{2}\left(\operatorname{dim} \mathfrak{g}+\operatorname{dim} \mathfrak{g}^{X}\right)$. If $X \in \mathcal{N}$ then if $\mu$ is an eigenvalue of $E_{\mathfrak{g}}$ on $\mathscr{D}^{\prime} \mathscr{O}_{X}\left(\mathfrak{g}_{o}\right)^{G_{0}}$ then $\mu \leq-\frac{1}{2}\left(\operatorname{dim} \mathfrak{g}+\operatorname{dim} \mathfrak{g}^{X}\right)$ and the eigenspace for $-\frac{1}{2}\left(\operatorname{dim} \mathfrak{g}+\operatorname{dim} \mathfrak{g}^{X}\right)$ has dimension at most 1 .

Proof. That $E_{\mathfrak{g}}$ acts semisimply has already been observed. We prove the first assertion by downward induction on $p$. If $p=r$ then $X_{p}=\{0\}$. Thus $\mathscr{D}_{\mathcal{N}_{r}}^{\prime}\left(\mathfrak{g}_{o}\right)^{G_{o}}=S(\mathfrak{g})^{G} \delta_{0}$ with $\delta_{0}$ the Dirac delta function at 0 . Since $E_{\mathfrak{g}} \delta_{0}=$ $-(\operatorname{dim} \mathfrak{g}) \delta_{0}$, the result follows in this case. Assume the result for $p>q$; we now prove it for $p=q$. Let $X=X_{q}$. Let $\{X, Y, H\}$ be an $s$-triple containing $X$. Set $V=\mathfrak{g}_{o}^{Y}$. As in [RRGI, 8.3.6, p. 299] we choose $U$ an open neighborhood of 0 in $V$ so that if $\Phi(g, Z)=g(X+Z), g \in G_{o}, Z \in U$, then $\Phi$ is a submersion of $G \times U$ onto an open subset $\Omega$ of $\mathfrak{g}_{o}$ such that:

(i) $\Omega \cap \mathscr{N}_{p}=\mathscr{O}_{p}$, and

(ii) $(X+\stackrel{p}{U}) \cap \mathscr{p}_{p}=\{X\}$.

Since $\Phi$ is a submersion, we may define, for $T \in \mathscr{D}_{\mathscr{N}_{p}}^{\prime}\left(\mathfrak{g}_{o}\right)^{G_{o}}, \Phi^{o}(T) \in$ $\mathscr{D}^{\prime}(U)$ with supp $\Phi^{o}(T)=\{0\}$ (see [RRGI, p. 301]). Let $-\mu_{1}, \ldots,-\mu_{d}$ be the eigenvalues of $a d H$ on $V$ counting multiplicities. Then we can choose linear coordinates on $V, y_{1}, \ldots, y_{d}$ such that

$$
\Phi^{o}\left(E_{\mathfrak{g}} T\right)=\left(\sum\left(\frac{1}{2} \mu_{i}+1\right) y_{i} \frac{\partial}{\partial y_{i}}\right) \Phi^{o}(T) .
$$

We note that $\sum\left(\mu_{i}+1\right)=\operatorname{dim} \mathfrak{g}$ and that $\operatorname{dim} V=\operatorname{dim} \mathfrak{g}^{X}$ by TDS theory. We also note that $\Phi^{o}(T) \in S\left(V_{C}\right) \delta_{V, 0}=\mathscr{D}\left(\delta_{V, 0}\right.$ the Dirac delta for $V$ supported at 0$)$. The eigenvalues of $\sum\left(\frac{1}{2} \mu_{i}+1\right) y_{i} \partial / \partial y_{i}$ on $\mathscr{D}$ are of the form $-\sum\left(\frac{1}{2} \mu_{i}+1\right) a_{i}$ with $a_{i}>0$, each has finite multiplicity and the eigenspace for $-\sum\left(\frac{1}{2} \mu_{i}+1\right)$ is $\mathbf{C} \delta_{V, 0}$. Now the observations using TDS theory imply that

$$
\sum\left(\frac{1}{2} \mu_{i}+1\right)=\frac{1}{2}\left(\operatorname{dim} \mathfrak{g}+\operatorname{dim} \mathfrak{g}^{X}\right)
$$

If $\Phi^{o}(T)=0$ then $T \in \mathscr{D}_{\mathscr{N}_{p+1}}^{\prime}\left(\mathfrak{g}_{o}\right)^{G_{o}}$. The first assertion of the lemma now follows. As for the second, the proof of the first part implies that if $T \in$ $\mathscr{D}^{\prime} \mathscr{\mathscr { O }}_{X}\left(\mathfrak{g}_{o}\right)^{G_{o}}$ is an eigenvector for $E_{\mathfrak{g}}$ with eigenvalue $-\frac{1}{2}\left(\operatorname{dim} \mathfrak{g}+\operatorname{dim} \mathfrak{g}^{X}\right)$ then 
$\Phi^{o}(T) \in \mathbf{C} \delta_{V, 0}$ and if $\Phi^{o}(T)=0$ then $T=0$. This proves the second assertion of the lemma.

If $X \in \mathfrak{g}_{o}$ then we recall that there is a canonical $G_{o}$ invariant measure (the Kostant-Kirillov measure) on $\mathscr{O}_{X}$ given as follows. If $y \in \mathscr{O}_{X}$ and if $u, v \in T\left(\mathscr{O}_{X}\right)_{y}$ with $u=[y, U], v=[y, V], U, V \in \mathfrak{g}_{o}$, then set $\omega_{y}(u, v)=$ $B(y,[U, V])$. Then $\omega$ defines a symplectic structure on $\mathscr{O}_{X}$ and $\nu_{X}=\omega^{m(X)}$ defines a volume form on $\mathscr{O}_{X}$. In [RR] it has been shown that if $X \in \mathscr{N}$ and $f \in C_{c}\left(\mathfrak{g}_{o}\right)$ then

$$
\int_{\mathscr{O}_{X}} f \nu_{X}=T_{X}(f)
$$

is defined by an absolutely convergent integral and that $T_{X}$ defines a $G_{o^{-}}$ invariant Radon measure on $\mathfrak{g}_{o}$. Thus $T_{X} \in \mathscr{D}_{\mathscr{N}}^{\prime}\left(\mathfrak{g}_{o}\right)^{G_{o}}$. Clearly, $\operatorname{supp} T_{X} \subset$ $\overline{\mathscr{O}_{X}}$. Theorem 6.1 implies that as a $\mathbf{D}(\mathfrak{h})^{W}$-module we have

$$
\mathbf{D}(\mathfrak{g})^{G} T_{X}=\bigoplus_{\lambda \in \widehat{W}} m_{X}(\lambda) \widetilde{V}^{\lambda}
$$

We have therefore assigned to each $X \in \mathcal{N}$ a function $m_{X}: \widehat{W} \rightarrow \mathbf{N}$. If $\lambda \in \widehat{W}$ then set $j(\lambda)=\min \left\{j \mid \operatorname{Hom}_{W}\left(V_{\lambda}, \mathscr{H}_{j}\right) \neq 0\right\}$. Put $j(X)=\min \left\{j(\lambda) \mid m_{X}(\lambda) \neq\right.$ $0\}$.

Theorem 6.3. If $X \in \mathscr{N}$ then $j(X)=\frac{1}{2}\left(\operatorname{dim} \mathfrak{g}^{X}-l\right)$. Furthermore, $\{\lambda \in$ $\left.\widehat{W} \mid m_{X}(\lambda)>0\right\}$ consists of one element, $\lambda_{X}$ with $m_{X}\left(\lambda_{X}\right)=1$, and

$$
\operatorname{dim} \operatorname{Hom}_{W}\left(V_{\lambda_{X}}, \mathscr{H}_{j(X)}\right)=1 .
$$

This result will take some preparation. We note that there are choices of invariant measures on $G_{o}$ and $G_{o}^{X}=\left\{\left.g \in G\right|_{g} X=X\right\}$ such that if $f \in \mathscr{C}_{c}\left(\mathfrak{g}_{o}\right)$ then

$$
T_{X}(f)=\int_{G_{o} / G_{o}^{X}} f(g X) d\left(g G_{o}^{X}\right) .
$$

If $h \in G_{o}$ and if $h X=c X$ for some $c \in \mathbf{R}$ then

$$
\int_{G_{o} / G_{o}^{X}} f(g h X) d\left(g G_{o}^{X}\right)=\operatorname{det}\left(h_{\mid g_{o}^{X}}\right)^{-1} \int_{G_{o} / G_{o}^{X}} f(g X) d\left(g G_{o}^{X}\right)
$$

for $f \in C_{c}\left(\mathfrak{g}_{o}\right)$.

As above, we will look upon the $\mathscr{A}$-module $\mathscr{A} T_{X}$ as a $\mathscr{B}$-module via $\delta$.

Lemma 6.4. Let $X \in \mathscr{N}$. Then $E_{\mathfrak{h}} T_{X}=-\frac{1}{2}\left(\operatorname{dim} \mathfrak{g}_{o}^{X}+l\right) T_{X}$.

Proof. We note that $E_{\mathfrak{g}} T_{x}(f)=T_{x}\left(e_{\mathfrak{g}}^{t} f\right)=-m T_{x}(f)-T_{X}\left(E_{\mathfrak{g}} f\right)$. If $X=0$ then $T_{0}$ is the Dirac delta function at 0 . Thus $T_{0}\left(E_{\mathrm{g}} f\right)=0$. Thus in this case $E_{\mathrm{g}} T_{0}=-m T_{0}$. Otherwise, let $\{X, Y, H\}$ be an $s$-triple containing $X$. Then

$$
f\left(g e^{(\log t) a d H / 2} X\right)=f(\operatorname{tg} X)
$$


for $g \in G_{o}, t>0$. Thus

$$
E_{\mathrm{g}} f(g X)=\frac{d}{d t}{ }_{\mid t=1} f\left(g e^{(\log t) a d H / 2} X\right) .
$$

Hence, if we apply the observation above, we have

$$
E_{\mathfrak{g}} T_{X}=\left(\frac{1}{2} \operatorname{tr} a d H_{\left.\right|_{\mathfrak{g}} ^{X}}-m\right) T_{X} .
$$

Let $\mathfrak{g}^{X}=\bigoplus \mathfrak{g}_{\lambda}^{X}$ with $\mathfrak{g}_{\lambda}^{X}$ the $\lambda$ eigenspace for ad $H$ in $\mathfrak{g}^{X}$. Then standard TDS theory implies that

$$
\sum_{\lambda} \operatorname{dim}\left(\mathfrak{g}_{\lambda}^{X}\right)(\lambda+1)=\operatorname{dim} \mathfrak{g}
$$

Hence

$$
\operatorname{tr} a d H_{\mid \mathfrak{g}}{ }^{x}=\operatorname{dim} \mathfrak{g}-\operatorname{dim} \mathfrak{g}^{X} .
$$

We have thus shown that

$$
E_{\mathfrak{g}} T_{X}=-\frac{1}{2}\left(\operatorname{dim} \mathfrak{g}+\operatorname{dim} \mathfrak{g}^{X}\right) T_{X} .
$$

This formula is valid if $X=0$.

We note as above that $\delta\left(E_{\mathfrak{g}}+\frac{m}{2}\right)=E_{\mathfrak{h}}+\frac{l}{2}$. Since $m=\operatorname{dim} \mathfrak{g}$, the lemma follows.

We will now give another interpretation of the $\mathbf{D}(\mathfrak{h})$-module, $S(\mathfrak{h})$. Let $\delta_{0} \in \mathscr{P}(\mathfrak{h})^{*}$ be defined by $\delta_{0}(f)=f(0)$. If $D \in \mathbf{D}(\mathfrak{h})$ and if $\lambda \in \mathscr{P}(\mathfrak{h})^{*}$ then set $D \lambda=\lambda \circ D^{T}$. Then our module structure on $S(\mathfrak{g})$ is just the module $D(\mathfrak{h}) \delta_{0}$.

Lemma 6.5. If $\lambda \in \widehat{W}$ then the highest eigenvalue of $E_{\mathfrak{h}}$ on $\widetilde{V}^{\lambda}$ is $-l-j(\lambda)$ and it has multiplicity $\operatorname{dim} \operatorname{Hom}_{W}\left(V_{\lambda}, \mathscr{H}_{j(\lambda)}\right)$.

Proof. We realize $\widetilde{V}^{\lambda}$ as $\mathscr{B} p \delta_{o}$ with $p \in \phi\left(\mathscr{H}_{j(\lambda)}[\lambda]\right)$. We note that

$$
E_{\mathfrak{h}} p \delta_{o}=(-l-j(\lambda)) p \delta_{o} .
$$

The lemma now follows from Theorems 1.6 and 2.7.

Lemma 6.6. If $X \in \mathscr{N}$ then the highest eigenvalue of $E_{\mathfrak{g}}$ on $\mathscr{A} T_{X}$ is $-\frac{1}{2}\left(\operatorname{dim} \mathfrak{g}+\operatorname{dim} \mathfrak{g}^{X}\right)$ and it occurs with multiplicity 1 .

Proof. In light of $(*)$ in the proof of Lemma 6.4, the eigenspace for $E_{\mathfrak{g}}$ with eigenvalue $-\frac{1}{2}\left(\operatorname{dim} \mathfrak{g}+\operatorname{dim} \mathfrak{g}^{X}\right)$ has positive dimension. The result now follows from the second assertion of Lemma 6.2.

Proof of Theorem 6.3. As a $\mathscr{B}$-module, $M=\mathscr{A} T_{X}$ is isomorphic with $\bigoplus_{\lambda \in \widehat{W}} m_{X}(\lambda) \tilde{V}^{\lambda}$. Thus the highest eigenvalue of $E_{\mathfrak{h}}$ on $M$ is $-l-j(X)$ with multiplicity equal to

$$
a=\sum_{\substack{\lambda \in \widehat{W} \\ j(\lambda)=j(X)}} \operatorname{dim} \operatorname{Hom}_{W}\left(V_{\lambda}, \mathscr{H}_{j(x)}\right) m_{X}(\lambda) .
$$


Lemma 6.3 in combination with Lemma 6.4 implies that $-l-j(X)=$ $-\frac{1}{2}\left(\operatorname{dim} \mathfrak{g}^{X}+l\right)$ and that $a=1$. Thus $j(X)=\frac{1}{2}\left(\operatorname{dim}_{\mathfrak{g}}{ }^{X}-l\right)$ and, since $a=1, \quad\left\{\lambda \in \widehat{W} \mid m_{X}(\lambda)>0, j(\lambda)=j(X)\right\}$ consists of one element $\lambda_{X}$ with $m_{X}\left(\lambda_{X}\right)=1$ and $\operatorname{dim} \operatorname{Hom}_{W}\left(V_{\lambda_{X}}, \mathscr{H}_{j(X)}\right)=1$. On the other hand, $M$ is generated by one element in the $-l-j(X)$ eigenspace of $M$. Since $M$ splits into a direct sum of modules isomorphic of the form $\widetilde{V}^{\lambda}$ and $m_{X}(\lambda)=0$ for $j(\lambda)<j(X), m_{X}(\lambda)=0$ if $j(\lambda)=j(X)$ and $\lambda \neq \lambda_{X}$, it follows that $M \cong \widetilde{V}^{\lambda_{X}}$. This completes the proof of the theorem.

Since $\mathscr{O}_{X}$ is a cone for $X \in \mathcal{N}, T_{X}$ is a homogeneous distribution on $\mathfrak{g}_{o}$. Hence $T_{X}$ is tempered. We will now analyze $\widehat{T}_{X}$. Choose $\mathfrak{h}$ such that $\mathfrak{h}_{o}=\mathfrak{h} \cap \mathfrak{g}_{o}$ is a real form of $\mathfrak{h}$. We note that $S_{+}(\mathfrak{g})^{G} \widehat{T}_{X}=0$. Thus $\widehat{T}_{X_{\mid \mathfrak{g}_{o} \cap \mathfrak{g}^{\prime}}}$ is a real analytic function which completely determines $T_{X}$ (cf. Theorem 5.3).

Theorem 6.7. Let $X \in \mathscr{N}$. If $C$ is a connected component of $\mathfrak{h}_{o} \cap \mathfrak{h}^{\prime}$ then there exists $h_{C} \in \mathscr{H}_{j(X)}\left[\lambda_{X}\right]$ such that $\widehat{T}_{X_{\mid C}}=h_{C} / \pi$.

Proof. If $f \in \mathscr{S}\left(\mathfrak{h}_{o}\right)$ then we define

$$
\mathscr{F} f(x)=\hat{f}(x)=\frac{1}{(2 \pi)^{l / 2}} \int_{\mathfrak{h}_{o}} f(y) e^{-i B(y, x)} d y .
$$

Here $d y$ is the Lebesgue measure on $\mathfrak{h}_{o}$ corresponding to a pseudo-orthonormal basis of $\mathfrak{h}_{o}$. If $T \in \mathscr{S}^{\prime}\left(\mathfrak{h}_{o}\right)$ and if $f \in \mathscr{P}_{j}(\mathfrak{h})$ (resp. $p \in S_{j}(\mathfrak{h})$ ) then $(f T)=$ $\phi(f) \hat{t}$ (resp. $(p t) \hat{t}=\phi(p) \hat{t}$, where $\phi$ is defined as in Appendix 1 corresponding to an orthonormal basis of $\mathfrak{h}$. Thus we have

(i) $\delta(\widehat{D})=\phi(\delta(D))$.

We also note that if $M$ is the $\mathscr{B}$-module $\widetilde{V}^{\lambda}$ with action given by $D \cdot m=$ $\widehat{D} m$ then $M \cong V^{\lambda}$. With these observations in hand we can prove the theorem.

Set $T=T_{X}$. As a $\mathscr{B}$-module $\mathscr{A} \widehat{T}$ is isomorphic with $V^{\lambda_{X}}$. Let $C$ be as in the statement. If $D \in \mathscr{A}$ then $r(D) \widehat{T}_{\mid C}=\pi^{-1} \delta(D) \pi \widehat{T}_{\mid C}$. Since $\mathscr{P}_{+}(\mathfrak{g})^{G} T=$ $0, S_{+}(\mathfrak{g})^{G} \widehat{T}=0$. Thus

$$
\pi r\left(S_{+}(\mathfrak{g})^{W}\right) \widehat{T}_{\mid C}=S_{+}(\mathfrak{h})^{W} \pi \widehat{T}_{\mid C}=0 .
$$

This implies that $\pi \widehat{T}_{\mid C}=h_{C}$ with $h_{C} \in \mathscr{H}$. Since $\widehat{E}_{\mathfrak{h}}=-E_{\mathfrak{h}}+l$, we see that $E_{\mathfrak{h}} H_{C}=j(X) h_{C}$. The above observations imply that $\mathbf{D}(\mathfrak{h})^{W} h_{C} \cong V^{\lambda_{X}}$ as a $\mathbf{D}(\mathfrak{h})^{W}$-module. Hence $h_{C} \in \mathscr{H}_{j(X)}\left[\lambda_{x}\right]$. This completes the proof.

We now look at a special case of these results. Let $\mathfrak{g}_{1}$ be a semisimple Lie algebra over $\mathbf{C}$. Let $\mathfrak{g}_{o}$ denote $\mathfrak{g}_{1}$ as a Lie algebra over $\mathbf{R}$. Let $\mathfrak{u}_{1}$ be a compact form of $\mathfrak{g}_{1}$. Let $\bar{X}$ denote complex conjugation of $X \in \mathfrak{g}_{1}$ with respect to $\mathfrak{u}_{1}$ Then we identify $\mathfrak{g}_{o}$ with the subalgebra $\left\{(X, \bar{X}) \in \mathfrak{g}_{1} \times \mathfrak{g}_{1} \mid X \in \mathfrak{g}_{1}\right\}$ of $\mathfrak{g}_{1} \times \mathfrak{g}_{1}$. If $\mathfrak{h}_{1}$ is a Cartan subalgebra of $\mathfrak{g}_{1}$ then $\mathfrak{h}_{o}=\left\{(X, \bar{X}) \mid X \in \mathfrak{h}_{1}\right\}$ is a Cartan subalgebra of $\mathfrak{g}_{o}$. With these identifications $\mathfrak{g}=\mathfrak{g}_{1} \times \mathfrak{g}_{1}$ and $\mathfrak{h}=\mathfrak{h}_{1} \times \mathfrak{h}_{1}$. 
$W(\mathfrak{g}, \mathfrak{h})=W\left(\mathfrak{g}_{1}, \mathfrak{h}_{1}\right) \times W\left(\mathfrak{g}_{1}, \mathfrak{h}_{1}\right)$. We set $W=W(\mathfrak{g}, \mathfrak{h}), W_{1}=W\left(\mathfrak{g}_{1}, \mathfrak{h}_{1}\right)$. Then $\left\{(s, s) \mid s \in W_{1}\right\}=\left\{s \in W \mid\right.$ there exists $g \in G_{o}$ such that $\left.g_{\mid \mathfrak{h}}=s\right\}$. If $\lambda, \mu \in \widehat{W}_{1}$ then we denote by $\lambda \widehat{\otimes} \mu$ their exterior tensor product as an element of $\widehat{W}$. We fix a system of positive roots, $P_{1}$, for the roots of $\mathfrak{g}_{1}$ with respect to $\mathfrak{h}_{1}$. Then $P=\left\{(\alpha, 0) \mid \alpha \in P_{1}\right\} \cup\left\{(0, \alpha) \mid \alpha \in P_{1}\right\}$ is a system of positive roots for $\mathfrak{g}$ with respect to $\mathfrak{h}$. This implies that if $\pi_{1}=\prod_{\alpha \in P_{1}} \alpha$ and $\pi=\Pi_{\alpha \in P} \alpha$ then

$$
\pi((X, \bar{X}))=\pi_{1}(X) \pi_{1}(\bar{X}) .
$$

We note that $\mathscr{A}(\mathfrak{g})=\mathscr{A}\left(\mathfrak{g}_{1}\right) \otimes \mathscr{A}\left(\mathfrak{g}_{1}\right)$ and $\mathscr{B}(\mathfrak{g}, h)=\mathscr{B}\left(\mathfrak{g}_{1}, \mathfrak{h}_{1}\right) \otimes \mathscr{B}\left(\mathfrak{g}_{1}, \mathfrak{h}_{1}\right)$. Thus the irreducible $\mathscr{B}(\mathfrak{g}, \mathfrak{h})$-module corresponding to $\lambda \otimes \mu$ in the category $\check{C}_{W}^{\prime}$ is $\widetilde{V}^{\lambda} \otimes \widetilde{V}^{\mu}$.

Theorem 6.8. If $X \in \mathfrak{g}_{1}$ is nilpotent then there exists $\sigma_{X} \in \widehat{W}_{1}$ such that $\lambda_{(X, \bar{X})}=\sigma_{X} \widehat{\otimes} \sigma_{X}$ (here $\left.\alpha \widehat{\otimes} \beta\left(s_{1}, s_{2}\right)=\alpha\left(s_{1}\right) \otimes \beta\left(s_{2}\right)\right)$ and $j\left(\sigma_{X}\right)=$ $\frac{1}{2}\left(\operatorname{dim} \mathfrak{g}_{1}^{X}-\operatorname{dim} \mathfrak{h}_{1}\right)$. Furthermore, if $X_{1}, X_{2}$ are nilpotent elements of $\mathfrak{g}_{1}$ and if $\sigma_{X_{1}}=\sigma_{X_{2}}$ then $G_{1} X_{1}=G_{1} X_{2}$.

Note. The above result implies that for a semisimple Lie algebra over $\mathbf{C}$, we have constructed an injective map from the set of nilpotent orbits of the adjoint group into the set of equivalence classes of irreducible representations of its Weyl group. At the end of this section we will use the results of [BV2, BV3, $\mathrm{HK}$ ] to show that this correspondence is the Springer correspondence [S].

Proof. Since $\mathfrak{h}_{o}-\left(\mathfrak{h}_{o} \cap \mathfrak{h}^{\prime}\right)$ is of (real) codimension 2 in $\mathfrak{h}_{o}$, we see that $\mathfrak{h}_{o} \cap \mathfrak{h}^{\prime}$ is connected. Set $T=T_{(X, \bar{X})}$ and $h_{\mathfrak{h}_{o} \cap \mathfrak{h}^{\prime}}=h_{X}$. If we had chosen a different Cartan subalgebra then it would be of the form $g \mathfrak{h}_{o}$ with $g \in G_{o}$. Since $T$ (hence $\widehat{T}$ ) is $G_{o}$-invariant, the corresponding " $h$ " would be given by $h(g H)=$ $h_{X}(H)$ for $H \in \mathfrak{h}_{o}$. Also by the $G_{o}$-invariance of $T$ and the $W_{o}$-invariance of $\pi$, we see that $w h_{X}=h_{X}$ for $w \in W_{o}$. This implies that $\left(V_{\lambda_{X}}\right)^{W_{o}} \neq 0$. Schur's Lemma, the fact that every irreducible representation of $W_{1}$ is defined over $\mathbf{R}$, and Theorem 6.3 imply that:

(i) $\lambda_{X}=\sigma_{X} \widehat{\otimes} \sigma_{X}$, and

(ii) $\operatorname{dim}\left(V_{\lambda_{X}}\right)^{W_{o}}=\operatorname{dim}\left(\mathscr{H}_{j(X, \bar{X})}\left[\lambda_{X}\right]\right)^{W_{o}}=1$.

This implies that if $X_{1}, X_{2}$ are nilpotent elements of $\mathfrak{g}_{1}$ and if $\lambda_{\left(X_{1}, \bar{X}_{1}\right)}=$ $\lambda_{\left(X_{2}, \bar{X}_{2}\right)}$ then there exists $c \in \mathbf{C}$ such that $\widehat{T}_{\left(X_{1}, \bar{X}_{1}\right)}=c \widehat{T}_{\left(X_{2}, \bar{X}_{2}\right)}$ on $\mathfrak{g}_{o}^{\prime}$ and, hence, on $\mathfrak{g}_{o}$ by Theorem 5.5. This implies that $T_{\left(X_{1}, \bar{X}_{1}\right)}=c T_{\left(X_{2}, \bar{X}_{2}\right)}$. Since $\mathscr{\theta}_{\left(X_{1}, \bar{X}_{1}\right)}$ is the unique open orbit in its closure, this completes the proof.

We now return to the general case. The main result in this section is

Theorem 6.9. Let $X \in \mathcal{N}$. If we look upon $X$ as a nilpotent element of $\mathfrak{g}$ then $\lambda_{X}=\sigma_{X}$.

Proof. We note that $G_{o} X$ is open in $G X \cap \mathfrak{g}_{o}$. We look upon $G X$ as a complex submanifold of $\mathfrak{g}$. By its very definition, $\nu_{X}$ extends to a holomorphic $2 m(X)$ form on $G X$. We will use the same symbol for this extension. Then up to 
a scalar multiple, $\nu_{(X, \bar{X})}=\nu_{X} \wedge \bar{\nu}_{X}$. Let $\mathscr{T}_{X}=\left\{f \in \mathscr{P}(\mathfrak{g}) \mid f\left(\mathscr{O}_{X}\right)=0\right\}$. If $x \in \mathscr{O}_{X}$ then there exists an open neighborhood, $\Omega_{o}$ of $x$ in $\mathfrak{g}_{o}$, and $f_{1}, \ldots, f_{m-2 m(X)} \in \mathscr{T}_{X}$ that are real valued on $\Omega_{o}$ and such that $\Omega_{o} \cap \mathscr{O}_{X}=$ $\left\{y \in \Omega_{o} \mid f_{i}(y)=0\right\}$. We may also assume that there is an open neighborhood, $\Omega$, of $G X$ in $\mathfrak{g}$ such that $\Omega \cap \mathfrak{g}_{o}=\Omega_{o}$ and $\Omega \cap G X=\left\{y \in \Omega \mid f_{i}(y)=0\right\}$. If we shrink $\Omega$, we may assume that $\Omega \cap G X$ is connected and there are local holomorphic coordinates $x_{1}, \ldots, x_{m}$ on $\Omega$ such that $x_{i}(x)=0$ and $x_{i+2 m(X)}=f_{i}$ for $i \geq 1$ and that $x_{1}, \ldots, x_{m}$ restricted to $\Omega_{o}$ are (real) local coordinates on $\Omega_{o}$. Furthermore, may assume that these coordinates on $\Omega_{o}$ satisfy the condition of Lemma 3 in Appendix 3 for $M=\mathscr{O}_{X}$ and that after reordering $\left\{\operatorname{Re} x_{1}, \ldots, \operatorname{Re} x_{m}, \operatorname{Im} x_{1}, \ldots, \operatorname{Im} x_{m}\right\}$ satisfy the conditions of Lemma 3 in Appendix 3 for $M=G X$. In the notation of Lemma 3 in Appendix 3, if $\eta_{o}$ is the " $\eta$ " for $\omega=\nu_{X}$ then $\eta_{o}$ extends to a holomorphic function on $G X \cap \Omega$ and the " $\eta$ " for $\nu_{(X, \bar{X})}$ is $\eta_{o} \bar{\eta}_{o}$.

If $D \in \mathbf{D}(\mathfrak{g})^{G}$ then we can think of $D$ as a holomorphic differential operator on $\mathfrak{g}$ and then we denote it by $D \otimes 1$. We can think of $\bar{D}$ as an antiholomorphic differential operator on $\mathfrak{g}$ and then we denote it by $1 \otimes \bar{D}$. Then $\mathbf{D}(\mathfrak{g} \times \mathfrak{g})^{G \times \bar{G}}$ is just $\mathbf{D}(\mathfrak{g})^{G} \otimes\left(\mathbf{D}(\mathfrak{g})^{G}\right)^{-}$. Also the corresponding " $\mathscr{A}$ " is $\mathscr{A} \otimes \overline{\mathscr{A}}$. Set $\mathscr{I}_{X}=$ $\left\{D \in \mathbf{D}(\mathfrak{g})^{G} \mid D T_{X}=0\right\}$; then Theorem 5.4 implies that $\mathscr{I}_{X} \supset \mathscr{I}$. The local criterion in Lemma 3 in Appendix 3 implies that if $D \in \mathscr{F}_{X}$ and if (in the notation of Appendix 3)

$$
D^{T}=\sum_{I, J} a_{I, J} \partial^{I} \partial^{J}
$$

then for each $J$ we have

$$
\sum_{I}(-1)^{|I|} \partial^{I}\left(\eta_{o} a_{I, J}\right)=0
$$

on $\Omega_{o} \cap \mathscr{O}_{X}$. Since all the terms extend to holomorphic functions on $\Omega \cap G X$, (1) is true on $\Omega \cap G X$. Now the local condition that $(D \otimes I) T=0$ is that for each $J$

$$
\sum_{I}(-1)^{|I|} \partial^{I}\left(\eta_{o} \bar{\eta}_{o} a_{I, J}\right)=0
$$

on $\Omega \cap G X$ with $\partial^{I}$ looked upon as partial derivatives from the holomorphic tangent space. Thus

$$
\sum_{I}(-1)^{|I|} \partial^{I}\left(\eta_{o} \bar{\eta}_{o} a_{I, J}\right)=\bar{\eta}_{o} \sum_{I}(-1)^{|I|} \partial^{I}\left(\eta_{o} a_{I, J}\right)=0
$$

Lemma 3 in Appendix 3 implies that $(D \otimes 1) T_{(X, \bar{X})}=0$. Similarly $(1 \otimes \bar{D}) T_{(X, \bar{X})}=0$. Thus the cyclic $\mathscr{A} \otimes \overline{\mathscr{A}}$-module generated by $T_{(X, \bar{X})}$ is a quotient of $\left(\mathscr{A} / \mathscr{I}_{X}\right) \otimes\left(\overline{\mathscr{A}} / \overline{\mathscr{I}_{X}}\right)$. But as a $\mathscr{B}$-module, $\mathscr{A} / \mathscr{I}_{X}$ is isomorphic with $\widehat{V}^{\lambda_{X}}$ (Theorem 3). This implies that $\widetilde{V}^{\sigma_{X}} \otimes \widetilde{V}^{\sigma_{X}}$ is isomorphic as a $\mathscr{B} \otimes \overline{\mathscr{B}}$-module with a quotient of $\widetilde{V}^{\lambda_{X}} \otimes \widetilde{V}^{\lambda_{X}}$. Thus $\lambda_{X} \otimes \lambda_{X}=\sigma_{X} \otimes \sigma_{X}$. Hence $\lambda_{X}=\sigma_{X}$.

We now close the circle of ideas that we have developed in this section by quoting an important theorem of [BV2; BV3; HK, Theorem 8.2, p. 357]. 
Theorem 6.10. If $\mathfrak{g}$ is a semisimple Lie algebra over $\mathbf{C}$ then the correspondence $\mathscr{O}_{X} \mapsto \sigma_{X}$ is the Springer correspondence.

Proof. In [HK] it is shown that $\left(T_{(X, \bar{X})} \hat{)}_{\mid \mathfrak{h}^{\prime}}=h /(\pi \otimes \bar{\pi})\right.$ and that $h$ is a $(W \times W)$-harmonic polynomial whose cyclic space under $W \times W$ is in the class of $\lambda \otimes \lambda$ with $\lambda$ the Springer representation associated with $X$. Theorem 6.7 now implies the result.

We conclude this section with a complete description of $\mathscr{D}_{\mathscr{N}}^{\prime}\left(\mathfrak{g}_{o}\right)^{G_{o}}$ as a $\mathscr{A}$ module in the special case when $\mathfrak{g}_{o}$ is a semisimple Lie algebra over $\mathbf{C}$ looked upon as a Lie algebra over $\mathbf{R}$. We use the notation and conventions established above for this special case.

Theorem 6.11. As a $\mathscr{B}(\mathfrak{g}, \mathfrak{h})$-module $\mathscr{D}_{\mathscr{N}}^{\prime}\left(\mathfrak{g}_{o}\right)^{G_{o}}$ is isomorphic with

$$
\bigoplus_{\lambda \in \widehat{W}_{1}} \widetilde{V}^{\lambda} \otimes \widetilde{V}^{\lambda}
$$

Proof. Theorem 6.1 implies that as a $\mathscr{B}$-module

$$
\mathscr{D}_{\mathcal{N}}^{\prime}\left(\mathfrak{g}_{o}\right)^{G_{o}} \simeq \bigoplus_{\lambda, \mu \in \widehat{W}_{1}} m_{\lambda, \mu} \widetilde{V}^{\lambda} \otimes \widetilde{V}^{\mu}
$$

Let $M$ be an irreducible nonzero $\mathscr{A}$-submodule of $\mathscr{D}_{\mathscr{N}}^{\prime}\left(\mathfrak{g}_{o}\right)^{G_{o}}$. Then since $\mathscr{P}_{+}(\mathfrak{g})^{G}$ acts locally nilpotently on $M$, there exists $T \neq 0, T \in M$ such that $\mathscr{P}_{+}(\mathfrak{g})^{W} T=0$. Thus $S_{+}(\mathfrak{g})^{G} \widehat{T}=0$. This implies that there exists $h \in$ $\mathscr{H}\left(\mathfrak{h}_{1}\right) \otimes \mathscr{H}\left(\mathfrak{h}_{1}\right)$ such that

$$
\widehat{T}(X, \bar{X})=h(X, \bar{X}) / \pi_{1}(X) \overline{\pi_{1}(X)}
$$

for $X \in \mathfrak{h}_{o} \cap \mathfrak{g}^{\prime}$. Furthermore, $\mathscr{B} h$ is irreducible and in $\check{\mathscr{C}}_{W}$. Thus, $\mathscr{B} h \simeq$ $V^{\lambda} \otimes V^{\mu}$ for some $\lambda, \mu \in \widehat{W}_{1}$. Hence, $h \in \mathscr{P}(\mathfrak{h})[\lambda \otimes \mu]$. But, if $s \in W_{1}$ then $\widehat{T}(s X, \overline{s X})=\widehat{T}(X, \bar{X})$ and $\pi_{1}(s X) \overline{\pi_{1}(s X)}=\pi(X) \overline{\pi(X)}$ for all $x \in \mathfrak{h}_{1}$. Thus relative to the diagonal action of $W_{1}$ on $V_{\lambda} \otimes V_{\mu}$ there is a nonzero fixed vector. This implies that $\lambda=\mu$. Let $W_{o}$ be the diagonal subgroup of $W_{1} \times W_{1}$. Then we have shown that $\pi \widehat{M}_{\mid \mathfrak{h}_{o} \cap \mathfrak{g}^{\prime}}$ is an irreducible $\mathscr{B}$-submodule of $\mathscr{P}(\mathfrak{h})[\lambda \otimes \lambda]^{W_{o}}$. The results of $\S 1$ easily imply that $\mathscr{P}(\mathfrak{h})[\lambda \otimes \lambda]^{W_{o}}$ is irreducible as a $\mathscr{B}$-module. We therefore see that $m_{\lambda, \mu} \leq 1$ and $m_{\lambda, \mu}=0$ if $\lambda \neq \mu$.

To prove that $m_{\lambda, \lambda}=1$, we will make use of Harish-Chandra's theory of orbital integrals. Let $\mathfrak{n}_{1}$ denote the sum of the root spaces in $\mathfrak{g}_{1}$ corresponding to $\alpha \in P_{1}$. Set $\mathfrak{n}=\left\{(X, \bar{X}) \mid X \in \mathfrak{n}_{1}\right\}$. Let, for $f \in \mathscr{S}\left(\mathfrak{g}_{o}\right), \Phi_{f}^{h_{o}}$ be as in [RRG1, 7.3.6]. There is a choice of Lebesgue measure on $\mathfrak{n}$ such that (see [RRG1, 7.3.8(4)]) if $H \in \mathfrak{h}_{o} \cap \mathfrak{g}^{\prime}$ then

$$
\Phi_{f}^{\mathfrak{h}_{o}}(H)=\int_{\mathfrak{n}} f^{o}(H+X) d X
$$

where if $K$ is the maximal compact subgroup of $G_{o}$ corresponding to $\mathfrak{u}$ and if $d k$ is normalized invariant measure on $K$ then

$$
f^{o}(X)=\int_{K} f(k X) d k
$$


This implies that if $f \in \mathscr{S}\left(\mathfrak{g}_{o}\right)$ then $\Phi_{f}^{\mathfrak{h}_{o}} \in \mathscr{S}\left(\mathfrak{h}_{o}\right)$. If $h \in \mathscr{H}(\mathfrak{h})^{W_{o}} \cap \mathscr{P}(\mathfrak{h})[\lambda \otimes \lambda]$ then we set

$$
S_{h}(f)=\int_{\mathfrak{h}_{o}} h(X, \bar{X}) \Phi_{f}^{\mathfrak{h}_{o}}(X) d X .
$$

Here we choose the Lebesgue measure on $\mathfrak{h}_{o}$ corresponding to a pseudo-orthonormal basis relative to the form $B$. If $p \in \mathscr{S}_{(\mathfrak{g})}{ }^{G}$ then

$$
\Phi_{p f}^{\mathfrak{h}_{o}}=\delta(p) \Phi_{f}^{\mathfrak{h}_{o}} .
$$

Thus if $D^{T}$ is the formal adjoint of $D \in \mathbf{D}\left(\mathfrak{h}_{o}\right)$ then $S_{h}(p f)=S_{\delta(p)^{T} h}(f)$ for $p \in S(\mathfrak{g})^{G}$. This implies that if we set $T_{h}(f)=S_{h}(\hat{f})$ then $\mathscr{P}_{+}(\mathfrak{g})^{G} T_{h}=0$. Hence supp $T_{h} \subset \mathscr{N} \cap \mathfrak{g}_{o}$.

Since $h(s X, \overline{s X})=h(X, \bar{X})$ for $X \in \mathfrak{h}_{1}$, we can define a $C^{\infty}$ function $\Psi_{h}$ on $\mathfrak{g}_{o} \cap \mathfrak{g}^{\prime}$ by $\Psi_{h}(g H)=h(H)$ for $H \in \mathfrak{h}_{o} \cap \mathfrak{g}^{\prime}$. Then noting that if $l_{1}=\operatorname{dim} \mathfrak{h}_{1}$ then $l=2 l_{1}$ and $d_{l}(X) \geq 0$ for $X \in \mathfrak{g}_{o}$, we have

$$
T_{h}(f)=\int_{\mathfrak{g}_{o} \cap \mathfrak{g}^{\prime}} \frac{\Psi_{h}(X)}{d_{2 l}(X)^{1 / 2}} \hat{f}(X) d X .
$$

Thus $T_{h} \in \mathscr{D}_{\mathscr{N}}^{\prime}\left(\mathfrak{g}_{o}\right)^{G_{o}}$. Now $\widehat{T}_{h}(f)=T_{h}\left(\mathscr{F}^{-1} f\right)=S_{h}\left(\mathscr{F}\left(\mathscr{F}^{-1} f\right)\right)=S_{h}(f)$. So $\widehat{T}_{h}=S_{h}$ and

$$
S_{h}(X)=\frac{h(X)}{\pi(X) \overline{\pi(X)}}
$$

for $X \in \mathfrak{h}_{o} \cap \mathfrak{g}^{\prime}$. In light of the material at the beginning of the proof of this theorem, it follows that $\mathscr{A} T_{h}$ is isomorphic with $\widetilde{V}^{\lambda} \otimes \widetilde{V}^{\lambda}$ when looked upon as a $B$-module.

\section{APPENDIX 1. POLYNOMIAL DIFFERENTIAL OPERATORS}

The purpose of this appendix is to compile basic theory of the Weyl algebra that will be used in the body of the paper. Let $V$ be an $n$-dimensional vector space over $\mathbf{C}$, and let $V^{*}$ denote the dual space. Let $\mathscr{P}(V)$ denote the algebra of all polynomials on $V$, and let $S(V)$ the symmetric algebra on $V \cdot \mathscr{P}^{k}(V)$ (resp. $S^{k}(V)$ ) will denote the space of elements in $\mathscr{P}(V)$ (resp. $S(V)$ ) homogeneous of degree $k$. We set $\mathscr{P}_{+}(V)=\sum_{k>0} \mathscr{P}^{k}(V), S_{+}(V)=\sum_{k>0} S^{k}(V)$. We also use the notation $\mathbf{D}(V)$ for the algebra of differential operators on $V$ with polynomial coefficients. If $v \in V$ then we look upon $v$ as a differential operator on $V$ using the operation $v f(x)=\partial_{v} f(x)=\frac{d}{d t} f(x+t v)_{\mid t=0}$ for $f \in \mathscr{P}(V)$. The corresponding map $v \mapsto \partial_{v}$ induces an injective algebra homomorphism of $S(V)$ into $\mathbf{D}(V)$ with image the algebra of constant coefficient differential operators on $V$. Thus if $u \in S(V)$, we will look upon $u$ as a constant coefficient differential operator on $V$. If $f \in \mathscr{P}(V)$ then we look upon $f$ as a differential operator on $V$ relative to polynomial multiplication. With these identifications the map $\mathscr{P}(V) \otimes S(V)$ to $\mathbf{D}(V)$ given by $f \otimes u \mapsto f u$ 
defines a linear bijection. If $v_{1}, \ldots, v_{n}$ is a basis of $V$ and if $x_{1}, \ldots x_{n}$ is the corresponding dual basis then we have identified $v_{i}$ with $\frac{\partial}{\partial x_{i}}$.

We use standard multi-index notation. If $I=\left(i_{1}, \ldots, i_{n}\right), i_{j} \in \mathbf{N}$ (the nonnegative integers), then we set $|I|=i_{1}+\cdots+i_{n}$. We write $x^{I}=x_{1}^{i_{1}} \cdots x_{n}^{i_{n}}$ and

$$
\partial^{I}=\frac{\partial^{|I|}}{\partial x_{1}^{i_{1}} \cdots \partial x_{n}^{i_{n}}} .
$$

We will also write $\partial_{i}=\frac{\partial}{\partial x_{i}}$. If $D \in \mathbf{D}(V)$ then $D=\sum_{|I|+|J| \leq k} a_{I, J} x^{I} \partial^{J}$ for some $k \in \mathbf{N}$ and $a_{I, J} \in \mathbf{C}$. The minimum of such $k$ is called the Bernstein degree of $D$. We set $\mathbf{D}^{k}(V)$ equal to space of all $D$ with Bernstein degree less than or equal to $k$. With this filtration $\mathbf{D}(V)$ is a filtered algebra with corresponding graded algebra, $\operatorname{Gr} \mathbf{D}(V)$, isomorphic with $\mathscr{P}\left(V \times V^{*}\right)=\mathscr{P}(V) \otimes$ $\mathscr{P}\left(V^{*}\right)$. If $\xi_{1}, \ldots, \xi_{n}$ are the dual linear coordinates on $V^{*}$ to $x_{1}, \ldots, x_{n}$ on $V$ (i.e., $\left.\xi_{i}(\lambda)=\lambda\left(v_{i}\right)\right)$ and if we set $\sigma_{k}(D)=\sum_{|I|+|J|=k} a_{I, J} x^{I} \xi^{J}$ then the map

$$
\sigma_{k}: \mathbf{D}^{k}(V) / \mathbf{D}^{k-1}(V) \rightarrow \mathscr{P}^{k}\left(V \times V^{*}\right)
$$

gives the isomorphism. We also note that if $D_{i} \in \mathbf{D}^{k_{i}}(V), i=1,2$, then $\left[D_{1}, D_{2}\right] \in \mathbf{D}^{k_{1}+k_{2}-2}(V)$. Furthermore,

$$
\sigma_{k_{1}+k_{2}-2}\left(\left[D_{1}, D_{2}\right]\right)=\left\{\sigma_{k_{1}}\left(D_{1}\right), \sigma_{k_{2}}\left(D_{2}\right)\right\}
$$

with $\{\ldots, \ldots\}$ the usual Poisson bracket given by

$$
\{f, g\}=\sum_{i}\left(\frac{\partial f}{\partial x_{i}} \frac{\partial g}{\partial \xi_{i}}-\frac{\partial f}{\partial \xi_{i}} \frac{\partial g}{\partial x_{i}}\right)
$$

for $f, g \in \mathscr{P}\left(V \times V^{*}\right)$.

We now record some (standard) results.

Proposition 1. $\mathbf{D}(V)$ is a simple algebra over $\mathbf{C}$.

For a proof see, for example, [Eh, Proposition 1.1].

If $M$ is a $\mathbf{D}(V)$-module with a filtration, $M_{0} \subset M_{1} \subset M_{2} \subset \cdots$ such that $\mathbf{D}^{i}(V) M_{j} \subset M_{j+i}$, then we say that $M$ is a filtered $\mathbf{D}(V)$-module. We note with the obvious action of $\mathbf{D}(V)$ on $\mathscr{P}(V)$ and the degree filtration that $\mathscr{P}(V)$ is an irreducible $\mathbf{D}(V)$-module.

Theorem 2. Suppose that $M$ is a filtered $\mathbf{D}(V)$-module with $\operatorname{dim} M_{q}<\infty$ and that $\operatorname{dim} M_{q} \leq c q^{p} / p$ ! for some $0<c<\infty$ and all $q$ sufficiently large. If $p<n$ then $M=0$ and if $p=n$ then $M$ has length at most $c$ as a $\mathbf{D}(V)$-module.

A proof of this result of J. Bernstein can be found in [Eh, Propositions 1.12 and 1.15].

The next theorem (an algebraic version of the Stone--Von Neumann theorem) is a special case of the Kashiwara Lemma of $\mathscr{D}$-module theory. Since it plays a critical role in this paper, we will record a relatively simple proof. 
Lemma 3. Let $M$ be a finitely generated $\mathbf{D}(V)$-module such that if $m \in M$ and $p \in S_{+}(V)$ then there exists $k$ such that $p^{k} m=0$. Then $M$ is $\mathbf{D}(V)$ isomorphic with a finite multiple of $\mathscr{P}(V)$. In particular, $M$ splits into a finite direct sum of irreducible $\mathbf{D}(V)$-modules.

Proof. Let $\mathscr{C}$ be the category of all $\mathbf{D}(V)$-modules $M$ satisfying the $S_{+}(V)$ nilpotence assumption of the lemma. We look upon $V$ as an abelian Lie algebra and $M$ as a $V$-module under the action $v \cdot m=\partial_{v} \cdot m$. We show that if $M \in \mathscr{C}$ then $H^{1}(V, M)=0$. Let $M^{k}=\left\{m \in M \mid S_{k}(V) m=0\right\}$. Then $M^{0}=\{0\}$ and $\bigcup_{k \geq 0} M^{k}=M$. Let $x_{1}, \ldots, x_{n}$ be linear coordinates on $V$. Put $E=\sum x_{i} \partial_{i}$. Then $E M^{k} \subset M^{k}$ for all $k$. Let $\omega \in Z^{1}(V, M)$; then there exists $k$ such that $\omega(V) \subset M^{k}$. Since $\omega \in Z^{1}(V, M), \partial_{i} \omega\left(\partial_{j}\right)=\partial_{j} \omega\left(\partial_{i}\right)$. Define $T(\omega)=\sum x_{i} \omega\left(\partial_{i}\right) \in M^{k+1}$. Then

$$
d T(\omega)\left(\partial_{j}\right)=\sum_{i} \partial_{j} x_{j} \omega\left(\partial_{i}\right)=\omega\left(\partial_{j}\right)+\sum_{i} x_{i} \partial_{j} \omega\left(\partial_{i}\right)=(E+1) \omega\left(\partial_{j}\right) .
$$

If $m \in M^{k}$ then we assert that $(E-k+1) m \in M^{k-1}$. Indeed, if $m \in M^{k}$ and $v \in S_{k-1}(V)$ then $v E m=[v, E] m+E v m$. The formula for $E$ implies that $E v m=0$. Now $[v, E]=(k-1) v$. Thus $v(E-k+1) m=0$. We therefore see that

$$
(d T(\omega)-k \omega)(V) \subset M^{k-1}
$$

Since $M^{0}=\{0\}$, this implies our vanishing assertion.

We note that $M^{1}=H^{0}(V, M)$. If $m \in M^{1}-\{0\}$ then $\mathbf{D}(V) m=\mathscr{P}(V) m$. Thus $\mathbf{D}(V) m$ is a nonzero quotient of the irreducible $\mathbf{D}(V)$-module, $\mathscr{P}(V)$. So it is equivalent with $\mathscr{P}(V)$. The above vanishing of first cohomology easily implies that, as a $\mathbf{D}(V)$-module, $M$ is isomorphic with $\mathscr{P}(V) \otimes M^{1}$ with $\mathbf{D}(V)$ acting on the first factor. This completes the proof.

We define an isomorphism, $\phi$, of $\mathbf{D}(V)$ by $\phi\left(x_{j}\right)=i \partial / \partial x_{j}$ and $\phi\left(\partial / \partial x_{j}\right)$ $=i x_{j}$. We note that $\phi$ depends on the choice of basis $\left\{x_{i}\right\}$ of $V^{*}$. If $\mathscr{B}$ is a subalgebra of $\mathbf{D}(V)$ such that $\phi(\mathscr{B})=\mathscr{B}$ and if $M$ is a $\mathscr{B}$-module then we denote by $\widetilde{M}$ the $\mathbf{D}(V)$-module with total space $M$ and with action $D \cdot m=\phi(D) m$.

Let $V_{o}$ be a real form of $V$, and let $B$ be a nondegenerate, symmetric, bilinear form on $V_{o}$. We will also denote by $B$ the complex bilinear extension of $B$ to $V$. Let $d x$ denote the Lebesgue measure on $V_{o}$ corresponding to a basis $\left\{v_{i}\right\}$ of $V_{o}$ such that $B\left(v_{i}, v_{j}\right)= \pm \delta_{i j}$. Let $\mathscr{S}\left(V_{o}\right)$ denote the Schwartz space of $V_{o}$. We use $B$ to define a Fourier transform $\mathscr{F}_{B}(f)=\hat{f}$ by

$$
\hat{f}(x)=\frac{1}{(2 \pi)^{n / 2}} \int_{V_{o}} f(y) e^{-i B(y, x)} d y
$$

If $U$ is an open subset of $V_{o}$ then we look upon $\mathbf{D}(V)$ as a subalgebra of the algebra of differential operators on $U$ with $C^{\infty}$ coefficients. If $\left\{x_{i}\right\}$ is defined as above with $\left\{v_{i}\right\}$ an orthonormal basis of $V$ then

$$
\mathscr{F}_{B}(D f)=\phi(D) \mathscr{F}_{B}(f)
$$




\section{APPENDIX 2. SOME INVARIANT THEORY}

Let $V_{o}$ be a vector space over $\mathbf{R}$ with inner product $(\ldots, \ldots)$. Let $\boldsymbol{\Phi}$ be a root system in $V_{o}$ (cf. [Bo]), and let $W$ denote the corresponding Weyl group. Let $V$ denote the complexification of $V_{o}$. We extend $(\ldots, \ldots)$ to a symmetric nondegenerate $\mathbf{C}$-bilinear form on $V$. We choose $u_{1}, \ldots, u_{n}$ to be basic (homogeneous) $W$-invariant polynomials in $\mathscr{P}(V)$ such that $u_{j}$ is real valued on $V_{o}$. Fix $\Phi^{+}$a system of positive roots in $\Phi$. Let $e_{1}, \ldots, e_{n}$ be an orthonormal basis of $V_{o}$, and let $x_{1}, \ldots, x_{n}$ be the corresponding linear coordinates on $V_{o}$ and $V$. Let $\xi_{1}, \ldots, \xi_{n}$ be the linear coordinates on $V_{o}^{*}$ corresponding to the dual basis to $e_{1}, \ldots, e_{n}$. We look upon $V^{*}$ as a $W$ module under the contragredient action and write $s \lambda=\lambda \circ s^{-1}$ for $\lambda \in V^{*}, s \in$ $W$. In this section we will be studying the action of $W$ on $V \times V^{*}$ given by $s(v, \lambda)=(s v, s \lambda)$.

We set $P=\sum_{i} \xi_{i} \partial / \partial x_{i}$. If $f \in \mathscr{P}(V)$ then $P^{k} f$ is called a polarization of $f$. We identify $\mathscr{P}(V)$ (resp. $\mathscr{P}\left(V^{*}\right)$ ) with the polynomials on $\mathscr{P}\left(V \times V^{*}\right)$ that depend only on $x_{1}, \ldots, x_{n}$ (resp. $\left.\xi_{1}, \ldots, \xi_{n}\right)$. The basic question of this section is to find a method of generating $\mathscr{P}\left(V \times V^{*}\right)^{W}$ using $\mathscr{P}(V)^{W}$. Based on a result in [W] for the symmetric group (and its easy extension to Weyl groups of type $\left.B_{n}=C_{n}\right)$ it has been suggested (sometimes conjectured) that $\mathscr{P}\left(V \times V^{*}\right)^{W}$ is generated as an algebra by the polarizations of the elements of $\mathscr{P}(V)^{W}$. We will call this suggestion the polarization hypothesis. Unfortunately, this hypothesis is false for Weyl groups of type $D_{n}$ for $n \geq 4$. Before we go on to positive results we give an example for $D_{4}$ of an invariant that is not in the algebra generated by polarizations of elements of $\mathscr{P}(V)^{W}$.

We will use the notation of [Bo]. Let $W$ be the Weyl group of type $D_{4}$, and use the coordinates $x_{i}=\varepsilon_{i}$. We take $u_{i}=\sum_{j} x_{j}^{2 i}$ for $i=1,2,3$ and $u_{4}=x_{1} x_{2} x_{3} x_{4}$. We note that, if $1 \leq i \leq 3, u_{i}$ is invariant under the bigger Weyl group, $W^{\prime}$, of $B_{4}$. Let $\omega \in W^{\prime}$ be the element such that $w \varepsilon_{i}=\varepsilon_{i}, i=$ $1,2,3$, and $w \varepsilon_{4}=-\varepsilon_{4}$. We set

$$
u=u(x, \xi)=\xi_{1}^{3} x_{2} x_{3} x_{4}+x_{1} \xi_{2}^{3} x_{3} x_{4}+x_{1} x_{2} \xi_{3}^{3} x_{4}+x_{1} x_{2} x_{3} \xi_{4}^{3}
$$

Then $u \in \mathscr{P}\left(V \times V^{*}\right)^{W}$. We assert that $u$ is not contained in the algebra generated by the polarizations of the elements of $\mathscr{P}(V)^{W}$. Since $P$ is a vector field it is enough to show that $u$ is not in the algebra generated by 1 and $P^{k} u_{j}$ for $k=1,2,3,4,5,6$ and $j=1,2,3,4$. So assume that $u$ is in this algebra. Since $w u=-u$, this implies (assuming the polarization hypothesis for $B_{4}$, which is correct) that

$$
u=f_{o} u_{4}+f_{1} P u_{4}+f_{2} P^{2} u_{4}+f_{3} P^{3} u_{4}+f_{4} P^{4} u_{4}
$$

with $f_{i}$ in the algebra generated by the polarizations of $u_{1}, u_{2}, u_{3}$ and $v=$ $\sum_{j} x_{j}^{8}$. We may assume that the $f_{i}$ are homogeneous in $x, \xi$. Bidegree considerations imply that $f_{o}$ and $f_{4}$ are 0 . Also, we may assume that $f_{i}$ has bidegree $(i-1,3-i$ ) (the first coordinate is the degree in $x$, the second is the degree 
in $\xi)$. Degree considerations imply that $f_{i}=c_{i} P^{3-i} u_{1}$. If we expand $u_{1} P^{3} u_{4}$ into monomials, we see that the term $x_{1}^{3} \xi_{2} \xi_{3} \xi_{4}$ occurs with a positive coefficient but cannot occur in the expansion of $u$ or $P^{3-i} u_{1} P^{i} u_{4}$ for $i=1,2$. Thus $f_{3}=0$. If we expand $P u_{1} P^{2} u_{4}$ then the monomial $x_{1}^{2} x_{2} \xi_{1} \xi_{3} \xi_{4}$ has a positive coefficient but cannot occur in $u$ or $P^{2} u_{1} P u_{4}$. Hence $f_{2}=0$. But now we have the contradiction that $u=c_{1} P^{2} u_{1} P u_{4}$.

Thus the polarization hypothesis is false in general. We now introduce a new hypothesis that we will prove all irreducible Weyl groups but $F_{4}, E_{6}, E_{7}, E_{8}$. We return to the general notation of this appendix.

Revised polarization hypothesis. Set

$$
P_{i}=\sum_{j} \frac{\partial u_{i}}{\partial \xi_{j}}(\xi) \frac{\partial}{\partial x_{i}}
$$

then $\mathscr{P}\left(V \times V^{*}\right)^{W}$ is generated as an algebra by 1 and the elements $P_{i_{1}} \cdots P_{i_{r}} u_{j}$, $r=0,1, \ldots, i_{k}=1, \ldots, n, j=1, \ldots, n$.

We state a simple lemma whose proof will be left to the reader.

Lemma 1. If the revised polarization hypothesis (resp. the polarization hypothesis) is true for every irreducible factor of $W$ then it is true for $W$. If after adjoining a variable $x_{n+1}$ and dual variable $\xi_{n+1}$ with $s x_{n+1}=x_{n+1}, s \in W$, the revised polarization hypothesis (resp. the polarization hypothesis) is true then it is true for $W$ on $V_{o}$.

Proposition 2. The polarization hypothesis is true for Weyl groups of type $A_{n}, B_{n}$ $=C_{n}, G_{2}$. The revised polarization hypothesis is true for $D_{n}, n \geq 4$.

Proof. We first consider the case of $G_{2}$. We note that the argument that we use would apply to any dihedral group. Let $\alpha_{1}, \alpha_{2}$ be simple roots for $\boldsymbol{\Phi}^{+}$. Set $x_{1}=\alpha_{1} /\left\|\alpha_{1}\right\|$, and take $e_{2}$ such that $\alpha_{1}\left(e_{2}\right)=0$. This defines $x_{1}, x_{2}$. Set $s_{1}=s_{\alpha_{1}}$. We take as basis generators for the invariants of $s_{1}, u=x_{1}^{2}+x_{2}^{2}$ and $x_{2}$. The polarization hypothesis is trivial for the group generated by $s_{1}$. Thus if $f \in \mathscr{P}\left(V \times V^{*}\right)^{W}$ then $f$ is a polynomial in $u, P u, x_{2}$, and $\xi_{2}$. We write $f=\sum_{p, q} \varphi_{p q}(u, P u) x_{2}^{p} \xi_{2}^{q}$. Let $\operatorname{Av}(g)=\frac{1}{|W|} \sum_{s \in W} s g$. Then since $s u=u$, $S P u=P u$ for $s \in W$,

$$
f=\operatorname{Av}(f)=\sum_{p, q} \varphi_{p, q}(u, P u) \operatorname{Av}\left(x_{2}^{p} \xi_{2}^{q}\right) .
$$

If $q=0$ then since $\operatorname{Av}\left(x_{2}^{p}\right)$ is $W$-invariant it is a polynomial in $u_{1}, u_{2}$. If $q>0$ then $g=\operatorname{Av}\left(x_{2}^{p+q}\right)$ is invariant, so it is a polynomial in $u_{1}, u_{2}$ and

$$
f=C \sum_{p, q} \varphi_{p, q}(u, P u) P^{q}(g)
$$

with $C^{-1}=(p+q)(p+q-1) \cdots(p+1)$.

We note that this argument is a variant of Weyl's original argument of $A_{n}$. We now consider the cases $A_{n-1}, B_{n}, D_{n}$. For all of these cases we take $x_{i}=\varepsilon_{i}$ 
as in [Bo]. We will use the symbol

$$
\left\langle x_{1}^{r_{1}} \xi_{1}^{s_{1}} \cdots x_{k}^{r_{k}} \xi_{k}^{s_{k}}\right\rangle=\frac{1}{n !} \sum_{w \in S_{n}} x_{w 1}^{r_{1}} \xi_{w 1}^{s_{1}} \cdots x_{w k}^{r_{k}} \xi_{w k}^{s_{k}}, \quad 1 \leq k \leq n .
$$

Then in the $A_{n-1}$ case every $f \in \mathscr{P}\left(V \times V^{*}\right)^{W}$ is a linear combination of terms as above. For $B_{n}$ the same is true as long as we restrict our symbols to $r_{i}+s_{i}$ even. For $D_{n}$ this is true if for $k<n$ the $r_{i}+s_{i}$ are even and for $k=n$ the $r_{i}+s_{i}$ are either all even or all odd.

Let $A$ be the algebra generated by 1 and the polarizations of the basic invariants. We show that the symbols above for $k<n$ are in $A$ by induction on $k$. If $k=1$ then

$$
\left(r_{1}+s_{1}\right) \cdots\left(r_{1}+1\right)\left\langle x_{1}^{r_{1}} \xi_{1}^{s_{1}}\right\rangle=P^{s_{1}}\left\langle x_{1}^{r_{1}+s_{1}}\right\rangle
$$

Assume the assertion for $1 \leq k-1<n$. If $k<n$ then $\left\langle x_{1}^{r_{1}} \xi_{1}^{s_{1}}\right\rangle\left\langle x_{2}^{r_{2}} \xi_{2}^{s_{2}} \cdots x_{k}^{r_{k}} \xi_{k}^{s_{k}}\right\rangle=\frac{n-k+1}{n}\left\langle x_{1}^{r_{1}} \xi_{1}^{s_{1}} \cdots x_{k}^{r_{k}} \xi_{k}^{s_{k}}\right\rangle+$ symbols with smaller $k$. For $A_{n-1}$ and $B_{n}$ this argument also works for $k=n$. This completes the proof for $A_{n-1}$ and $B_{n}$. If $k=n$ in the case of $D_{n}$ and if all of the $r_{i}+s_{i}$ are even then the same argument applies. We are thus left with the case when $r_{i}+s_{i}$ is odd for all $i=1, \ldots, n$, and we may assume the result for $B_{n}$.

We set $L_{2 j-1}=\sum_{i} \xi_{i}^{2 k-1} \partial / \partial x_{i}$. We note that $\sum C L_{2 k-1} \subset \sum_{j \leq n} \mathscr{P}\left(V^{*}\right)^{W} P_{j}$. (Here we take $u_{j}=\sum_{i} x_{i}^{2 j}$ for $j=1, \ldots, n-1$ and $u_{n}=x_{1} x_{2} \cdots x_{n}$. ) Indeed if $v_{k}(\xi)=\sum_{i} \xi_{i}^{2 k}$ then

$$
2 k L_{2 k-1}=\sum_{i} \frac{\partial}{\partial \xi_{i}} v_{k}(\xi) \frac{\partial}{\partial x_{i}} .
$$

Since $v_{k}$ is a polynomial in $u_{1}, \ldots, u_{n}$, the assertion follows from the chain rule. We now prove that if $A^{1}$ is the algebra generated by the polynomials $L_{j_{1}} L_{j_{2}} \cdots L_{j_{r}} f$ with $j_{i}$ odd and $f \in \mathscr{P}(V)^{W}$ then

$$
u=\left\langle x_{1}^{r_{1}} \xi_{1}^{s_{1}} \cdots x_{n}^{r_{n}} \xi_{n}^{s_{n}}\right\rangle \in A^{1}
$$

by induction on the number, $q$, of $s_{J} \neq 0$. If $q=0$, then $u \in \mathscr{P}(V)^{W} \subset A^{1}$. Assume $0 \leq q \leq k-1$. We now consider the case when $q=k$. Set $p=n-k$. Then we may (after relabeling) assume that

$$
u=\left\langle x_{1}^{r_{1}} \cdots x_{p}^{r_{p}} x_{p+1}^{r_{p+1}} \xi_{p+1}^{s_{p+1}} \cdots x_{n}^{r_{n}} \xi_{n}^{s_{n}}\right\rangle
$$

If all of the $r_{j}>0$ then

$$
u=x_{1} \cdots x_{n}\left\langle x_{1}^{r_{1}-1} \cdots x_{p}^{r_{p}-1} x_{p+1}^{r_{p+1}-1} \xi_{p+1}^{s_{p+1}} \cdots x_{n}^{r_{n}-1} \xi_{n}^{s_{n}}\right\rangle .
$$

The result for $B_{n}$ now implies that $u \in A$. Thus we may assume that $r_{p+1}=0$. Consider

$$
\begin{aligned}
L_{s_{p+1}} & \left\langle x_{1}^{r_{1}} \cdots x_{p}^{r_{p}} x_{p+1} x_{p+2}^{r_{p+2}} \xi_{P+2}^{s_{p+2}} \cdots x_{n}^{r_{n}} \xi_{n}^{s_{n}}\right\rangle \\
& =\sum_{j=1}^{p} r_{j}\left\langle x_{1}^{r_{1}} \cdots x_{j}^{r_{j}-1} \xi_{j}^{s_{p+1}} \cdots x_{p}^{r_{p}} x_{p+1} \cdots x_{n}^{r_{n}} \xi_{n}^{s_{n}}\right\rangle+u+v
\end{aligned}
$$


where $v$ is a sum of symbols with at most $q-1$ nonzero exponents for the $\xi_{i}$. We note that all of the terms in the first sum have at least one pair $\left(r_{i}, s_{i}\right)=$ $(1,0)$.

Thus we may assume that

$$
u=\left\langle x_{1} x_{2}^{r_{2}} \cdots x_{p}^{r_{p}} \xi_{p+1}^{s_{p+1}} \cdots x_{n}^{r_{n}} \xi_{n}^{s_{n}}\right\rangle .
$$

If we now repeat the above argument then

$$
\begin{aligned}
L_{s_{p+1}} & \left\langle x_{1} \cdots x_{p}^{r_{p}} x_{p+1} x_{p+2}^{r_{p+2}} \xi_{P+2}^{s_{p+2}} \cdots x_{n}^{r_{n}} \xi_{n}^{s_{n}}\right\rangle \\
& =\sum_{j=2}^{p} r_{j}\left\langle x_{1} \cdots x_{j}^{r_{j}-1} \xi_{j}^{s_{p+1}} \cdots x_{p}^{r_{p}} x_{p+1} \cdots x_{n}^{r_{n}} \xi_{n}^{s_{n}}\right\rangle+2 u+v
\end{aligned}
$$

with $v$ in $A^{1}$ (smaller number of nonzero $\xi_{i}$ exponents) and each term having two exponents $(1,0)$. If we continue in this way, we may assume that

$$
u=\left\langle x_{1} x_{2} \cdots x_{p} \xi_{p+1}^{s_{p+1}} \cdots x_{n}^{r_{n}} \xi_{n}^{s_{n}}\right\rangle
$$

Now

$$
L_{s_{p+1}}\left\langle x_{1} x_{2} \cdots x_{p} x_{p+1} \cdots x_{n}^{r_{n}} \xi_{n}^{s_{n}}\right\rangle=(p+1) u+v
$$

with $v \in A^{1}$. This completes the proof.

\section{APPENDIX 3. SOME OBSERVATIONS ABOUT DisTRIBUTIONS}

In this appendix we first give a slight generalization of the well-known result that a homogeneous distribution on $\mathbf{R}^{n}$ is tempered. We use it to show that certain distributions, locally defined, on a real semisimple Lie algebra extend to tempered distributions. If $\Omega$ is a compact subset of $\mathbf{R}^{n}$ then we set $C_{\Omega}^{\infty}\left(\mathbf{R}^{n}\right)$ equal to the space of all smooth functions on $\mathbf{R}^{n}$ such that supp $f \subset \Omega$ with the topology given by the seminorms $q_{I}(f)=\sup _{x \in \Omega}\left|\partial^{I} f(x)\right|$ for $I=$ $\left(i_{1}, \ldots, i_{n}\right), i_{j} \in \mathbf{N}(=\{0,1,2, \ldots\})$. Here we use standard multi-index notation as in Appendix 1.

As usual, a distribution on $\mathbf{R}^{n}$ is a linear function, $T$, on $C_{c}^{\infty}\left(\mathbf{R}^{n}\right)$ such that $T$ is continuous on each space $C_{\Omega}^{\infty}\left(\mathbf{R}^{n}\right)$. As a customary, we set $\mathscr{D}^{\prime}\left(\mathbf{R}^{n}\right)$ equal to the space of all distributions on $\mathbf{R}^{n}$. If $f \in C_{1}^{\infty}\left(\mathbf{R}^{n}\right)$ then we set

$$
p_{k, I}(f)=\sup _{x \in \mathbf{R}^{n}}\|x\|^{k}\left|\partial^{I} f(x)\right| \text {. }
$$

Here $\|x\|^{2}=x_{1}^{2}+\cdots+x_{n}^{2}$ (as usual) and $k \geq 0$. As is usual, we denote by $\mathscr{S}\left(\mathbf{R}^{n}\right)$ the space of all $f \in C^{\infty}\left(\mathbf{R}^{n}\right)$ such that $p_{k, I}(f)<\infty$ for all $k \geq$ $0, I$ endowed with the topology induced by the seminorms $p_{k, I}$. If $T \in$ $\mathscr{D}^{\prime}\left(\mathbf{R}^{n}\right)$ then (as usual) we say that $T$ is tempered if $T$ extends to a continuous functional on $\mathscr{S}\left(\mathbf{R}^{n}\right)$.

We set $E=\sum_{i} x_{i} \partial / \partial x_{i}$. The following is a mild extension of a well-known result and is no doubt also well known. The proof involves standard methods of distribution theory. 
Lemma 1. If $T \in \mathscr{D}^{\prime}\left(\mathbf{R}^{n}\right)$ is such that $\operatorname{dim} \mathbf{C}[E] T<\infty$ then $T$ is tempered.

We will now apply this lemma to the case of interest in this paper. Let $G$ be a semisimple group of inner type (cf. [RRGI, 2.2.8]). Let $\mathfrak{g}$ be the Lie algebra of $G$, and let $\theta$ be a Cartan involution of $G$. As usual, $B$ will denote the Killing form of $\mathfrak{g}$. We note that $\langle X, Y\rangle=-B(\theta X, Y)$ defines an inner product on $\mathfrak{g}$. Using an orthonormal basis of $\mathfrak{g}$ with respect to $\langle\ldots, \ldots\rangle$ we may identify $\mathfrak{g}$ with $\mathbf{R}^{n}$ with $n=\operatorname{dim} \mathfrak{g}$. If $X \in \mathfrak{g}$ then $X$ is said to be nilpotent if $\operatorname{Ad} X$ is nilpotent. Let $\mathscr{N}$ denote the variety of nilpotent elements of $\mathfrak{g}$. If $f \in C^{\infty}(\Omega)$ and if $g \in G$ then we set $\tau(g) f(X)=f\left(\operatorname{Ad}(g)^{-1} X\right)$. We set $\mathscr{D}^{\prime}(\Omega){ }^{G}=\left\{T \in \mathscr{D}^{\prime}(\Omega) \mid T(\tau(g) f)=T(f)\right.$ for all $\left.g \in G, f \in C_{c}^{\infty}(\Omega)\right\}$. The following result is due to Harish-Chandra. However, his proof is quite complicated (see [Var, pp. 128-137]).

Lemma 2. Let $\Omega$ be open in $\mathfrak{g}$ and completely invariant (see $\S 5$ ). If $\Omega \cap \mathscr{N} \neq \varnothing$ then $\mathscr{N} \subset \Omega$. If $T \in \mathscr{D}^{\prime}(\Omega)^{G}$ and if $\operatorname{supp} T \subset \mathscr{N}$ then $T$ extends to a tempered distribution on $\mathfrak{g}$. That is, there exists a tempered distribution $S$ on $\mathfrak{g}$ such that $T(f)=S(f)$ for $f \in C_{c}^{\infty}(\Omega)$.

Proof. If $\Omega \cap \mathscr{N} \neq \varnothing$ then, if $X \in \Omega \cap \mathscr{N}, X_{s}=0 \in \Omega$. If $X \in \mathscr{N}$ and if $\{X, Y, H\}$ is an $s$-triple in $\mathfrak{g}$ then $e^{-t a d H} X=e^{-2 t} X$. Thus if $t$ is sufficiently large $e^{-t a d H} X \in \Omega$. Hence $X \in \Omega$. According to [Var, Theorem 28, p. 19] there exists $\alpha \in C^{\infty}(\Omega)^{G}$ such that $\operatorname{supp} \alpha \subset \Omega$, and $\alpha$ is identically equal to 1 in a neighborhood of 0 . Thus if $X \in \mathscr{N}$ then $\alpha(X)=\alpha\left(e^{-t a d H} X\right)=\lim _{t \rightarrow+\infty} \alpha\left(e^{-t a d H} X\right)=\alpha(0)=1$. Thus $\alpha$ is identically equal to 1 in a neighborhood of $\mathscr{N}$. We set $S=\alpha T$. Then $S \in \mathscr{D}^{\prime}(\mathfrak{g})^{G}$ and $S(f)=T(f)$ for $f \in C_{c}^{\infty}(\Omega)$. Thus to prove the lemma it is enough to show that $S$ is tempered. We note that the support of $S$ is contained in $\mathscr{N}$. Hence Lemma 8.3.7 in [RRGI] implies that $\operatorname{dim} \mathrm{C}[E] S<\infty$. Lemma 1 implies that $S$ is tempered.

We will now prove another technical result that will be used in $\S 7$. Let $M$ be an $m$-dimensional submanifold of $\mathbf{R}^{n}$. Assume that for each $u \in M$ there exists a neighborhood, $\Omega$, of $u$ in $\mathbf{R}^{n}$ and local coordinates $\left\{x_{1}, \ldots, x_{n}\right\}$ on $\Omega$ such that $x_{i}(u)=0,1 \leq i \leq n$, and $M \cap \Omega=\left\{y \in \Omega \mid x_{i}(y)=0, i>m\right\}$. Let $\omega$ be a smooth $d$-form on $M$ that defines a volume form on $M$. We define a distribution on $\mathbf{R}^{n}$ by $T(f)=\int_{M} f \omega, f \in C_{c}^{\infty}\left(\mathbf{R}^{n}\right)$ (note that our local condition implies that $T$ is in fact a Radon measure on $\mathbf{R}^{n}$ ). If $D$ is a differential operator on $\mathbf{R}^{n}$ then let $D^{T}$ denote its formal adjoint with respect to Lebesgue measure.

Lemma 3. Let $D$ be a differential operator on $\mathbf{R}^{n}$ such that $D T=0$. Let $u \in M$, and let $\Omega$ and $x_{1}, \ldots, x_{n}$ be as above. Define $\eta \in C^{\infty}(\Omega \cap M)$ by

$$
\omega_{\mid \Omega \cap M}=\eta d x_{1} \wedge \cdots \wedge d x_{m}
$$

Write $D^{T}=\sum_{I, J} a_{I, J} \partial^{I} \partial^{J}$ with the "I" multi-indices of the form $\left(i_{1}, \ldots, i_{m}\right.$, 0 ) and the " $J$ " multi-indices of the form $\left(0, j_{m+1}, \ldots, j_{n}\right)$. Then for each $J$ 
we have

$$
\sum_{I}(-1)^{|I|} \partial^{I}\left(\eta a_{I, J}\right)(u)=0 .
$$

Furthermore, if $D$ is a differential operator on $\mathbf{R}^{n}$ and $D$ satisfies all of these local conditions then $D T=0$.

Proof. Assume that $D$ is of order $k$. Let $\varphi \in C_{c}^{\infty}(\Omega)$ be such that the support of $\varphi$ is contained in the set $R=\left\{y \in \Omega|| x_{i}(y) \mid<r, 1 \leq i \leq n\right\}$. Let $V=\left\{y \in \Omega\left|x_{i}(y)=0, i>m,\right| x_{i}(y) \mid<r, \quad 1 \leq i \leq m\right\}$. Then

$$
\begin{aligned}
D T(\varphi) & =\sum_{I, J} \int_{V} \eta a_{I, J}\left(\partial^{I} \partial^{J} \varphi\right) d x_{1} \wedge \cdots \wedge d x_{m} \\
& =\sum_{I, J}(-1)^{|I|} \int_{V} \partial^{I}\left(\eta a_{I, J}\right) \partial^{J} \varphi d x_{1} \wedge \cdots \wedge d x_{m}
\end{aligned}
$$

By a simple iteration of [Ho, Theorem 1.2.6], if $\psi \in C_{c}^{\infty}(V)$ then then for each $J=\left(0, j_{m+1}, \ldots, j_{n}\right)$ with $|J| \leq k$ there exists $\varphi \in C_{c}^{\infty}(R)$ such that $\partial^{K} \varphi_{\mid V}=\delta_{J, K} \psi$ for all $K=\left(0, k_{m+1}, \ldots, k_{n}\right)$ with $|K| \leq k$. This clearly implies the first assertion. The converse also follows from the above formula.

\section{NOTE ADDED IN PROOF}

Lance Small has observed that Lemma 1.2 can be deduced from the results in S. Montgomery, Fixed rings of finite automorphism groups of associative rings, Lecture Notes in Math., vol. 818, Springer, Berlin, 1980.

\section{REFERENCES}

[BV1] D. Barbasch and D. Vogan, The local structure of characters, J. Funct. Anal. 37 (1980), 27-55.

[BV2] _ Primitive ideals and orbital integrals in complex classical groups, Math. Ann. 259 (1982), 153-199.

[BV3] _ Primitive ideals and orbital integrals in complex exceptional groups, J. Algebra 80 (1983), 350-382.

[B] J. N. Bernstein, The analytic continuation of generalized functions with respect to a parameter, Funct. Anal. Appl. 6 (1972), 26-40.

[Bo] N. Bourbaki, Groupes et Algèbras de Lie, Chapitres IV, V, et VI, Hermann, Paris, 1968.

[Eh] F. Ehlers, The Weyl algebra, Algebraic D-modules, Chapter V, Perspect. Math., vol. 2, Academic Press, Boston, MA, 1987.

[H1] Harish-Chandra, Differential operators on a semisimple Lie algebra, Amer. J. Math. 79 (1957), 87-120.

[H2] Invariant eigendistributions on a semisimple Lie algebra, Inst. Hautes Études Sci. Publ. Math. 27 (1965), 5-54.

[H3] Invariant differential operators and distributions on a semisimple Lie algebra, Amer. J. Math. 86 (1964), 534-564.

[Ho] L. Hörmander, The analysis of linear partial differential operators. I, Springer-Verlag, Berlin, 1990.

[HK] R. Hotta and M. Kashiwara, The invariant holonomic system on a semisimple Lie algebra, Invent. Math. 75 (1984), 327-358. 
[K] B. Kostant, Lie group representations on polynomial rings, Amer. J. Math. 85 (1963), 327- 404.

[RR] R. Ranga Rao, Orbital integrals in reductive groups, Ann. of Math. (2) 96 (1972), 505-510.

[S] T. A. Springer, Trigonometric sums, Green functions of finite groups and representations of Weyl groups, Invent. Math. 36 (1976), 209-224.

[Var] V. S. Varadarajan, Harmonic analysis on real reductive groups, Lecture Notes in Math., vol. 576, Springer-Verlag, Berlin, 1977.

[RRGI] N. R. Wallach, Real reductive groups. I, Academic Press, Boston, MA, 1988.

[W] H. Weyl, The classical groups, their invariants and representations, 2nd. ed., Princeton University Press, Princeton, NJ, 1949.

Department of Mathematics, University of California, San Diego, la Jolla, CALIFORNIA 92093-0001

E-mail address: nwallach@ math.ucsd.edu 